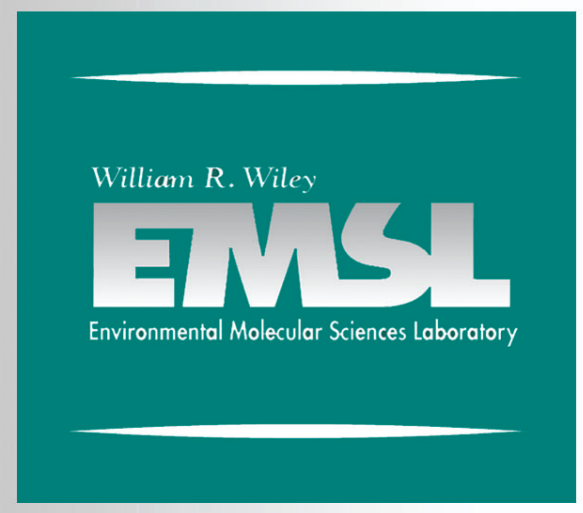

\title{
Mechanistic Process Modeling for Subsurface Remediation
}

\author{
S.B. Yabusaki \\ S.L. Bryant \\ S. Chen \\ G.I. Fann \\ G.P. Flach \\ W.G. Gray \\ J.P. Holland \\ K.E. Jordan \\ R.S. Maier
}

J. Malard

D.R. Rector

W.E. Soll

C.I. Steefel

A.F.B. Tompson

H.E. Trease

M.F. Wheeler

B.D. Wood

August 2002

Theory, Modeling

\section{and Simulation}

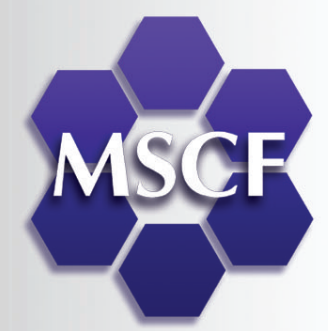


This research was performed in part using the Molecular Science Computing Facility (MSCF) in the William R. Wiley Environmental Molecular Sciences Laboratory, a national scientific user facility sponsored by the U.S. Department of Energy's Office of Biological and Environmental Research and located at the Pacific Northwest National Laboratory. Pacific Northwest is operated for the Department of Energy by Battelle.

\title{
DISCLAIMER
}

This report was prepared as an account of work sponsored by an agency of the United States Government. Neither the United States Government nor any agency thereof, nor Battelle Memorial Institute, nor any of their employees, makes any warranty, express or implied, or assumes any legal liability or responsibility for the accuracy, completeness, or usefulness of any information, apparatus, product, or process disclosed, or represents that its use would not infringe privately owned rights. Reference herein to any specific commercial product, process, or service by trade name, trademark, manufacturer, or otherwise does not necessarily constitute or imply its endorsement, recommendation, or favoring by the United States Government or any agency thereof, or Battelle Memorial Institute. The views and opinions of authors expressed herein do not necessarily state or reflect those of the United States Government or any agency thereof.

\author{
PACIFIC NORTHWEST NATIONAL LABORATORY \\ operated by \\ BATTELLE \\ for the \\ UNITED STATES DEPARTMENT OF ENERGY \\ under Contract DE-ACO6-76RLO183O
}

http://www.pnl.gov/main/publications/external/technical_reports/pnnl-14011.pdf

PNNL-14011, Published by Pacific Northwest National Laboratory for the

Environmental Molecular Sciences Laboratory 


\title{
Mechanistic Process \\ Modeling for \\ Subsurface Remediation
}

\author{
S. Yabusaki J. Malard \\ S.L. Bryant D.R. Rector \\ S. Chen W.E. Soll \\ G.I. Fann C.I. Steefel \\ G.P. Flach A.F.B. Tompson \\ W.G. Gray H.E. Trease \\ J.P. Holland M.F. Wheeler \\ K.E. Jordan B.D. Wood \\ R.S. Maier
}

August 2002

Published by Pacific Northwest National Laboratory for the Environmental Molecular Sciences Laboratory 


\section{Mechanistic Process Modeling for Subsurface Remediation}

\section{$\underline{\text { Research Team Members }}$}

Gregory P. Flach (DOE-EM)

Savannah River Technology Center

Jeffery P. Holland (DOD, SERDP)

Corps of Engineers Waterways Experiment Station

Robert S. Maier (DOD, SERDP)

Army High Performance Computing Research Center

Kirk E. Jordan (Industrial)

IBM T.J. Watson Research Center

Steven L. Bryant (NSF), Mary F. Wheeler (NSF)

University of Texas, Austin

George I. Fann (DOE: LTP, SC, LDRD), Joel Malard (DOE: SC, LDRD), David R. Rector (DOE: SC, EM, LDRD), Harold Trease (DOE: SC, LDRD), Brian D. Wood (DOE: SC, LDRD), Steve Yabusaki (DOE: SC, EM, EMSP, LDRD_

Pacific Northwest National Laboratory

Andrew F. B. Tompson (DOE: EMSP), Carl I. Steefel (DOE: EM)

Lawrence Livermore National Laboratory

Wendy E. Soll (DOE: EMSP), Shi-yi Chen (DOE: EMSP)

Los Alamos National Laboratory

William G. Gray (DOE: EMSP)

University of Notre Dame

Number of Hours Allocated for the Past Three Years (Exclude Refund Deposits)

Number of Hours Actually Used in the Past Three Year (Exclude Refunded Amounts)

\section{Overview of the Past Three Year's Accomplishments and Activities}

New partnerships were established in this project between PNNL, LANL, LLNL, SRTC, University of Texas, University of Notre Dame, Army High Performance Computing Research Center, and the Army Engineers Waterways Experiment Station. The goal of this project was to develop the first generation of models that fully address the coupling of dominant processes controlling the behavior of fluid, chemical and biological components in the subsurface. The large memory and computational performance of multiprocessor computing architectures would be exploited to provide modeling capabilities with unprecedented process detail and resolution to 
assess new scientific hypotheses, assist with experimental design, and to evaluate environmental technologies and remediation design. Moreover, the proposed capability developments would advance the scientific agenda for the subsurface through the realized advances in complex multiple-phase reaction modeling.

One fundamental scientific advancement supported by the GC4 grant is the refinement of the governing equations for multiphase fluid flow at the Darcy continuum scale. This work, led by LANL, LLNL, and the University of Notre Dame, is worked to identify the mathematical description of the critical equations, terms, and parameters needed to accurately simulate multiphase flow. At the microscale, small scale flow in explicitly modeled pores were simulated with a lattice Boltzmann technique. These simulations on simplified and geometrically constrained porous systems were used to refine the continuum theory.

An important technical outcome of the GC4 team is a coupled multiphase flow and reactive transport simulation capability. The sophistication of this approach is directly applicable to fundamental science as well as engineering needs at DOE cleanup sites. Recent simulations of the behavior of leaks from a HLW tank at Hanford have demonstrated the utility of this effort. The developed codes address the impact of highly resolved depictions of physical and chemical heterogeneities on subsurface flow, transport, and reactions through the use of massively parallel processing computers.

Several important supporting technologies have also been developed on the project. The parallel Fortran preprocessor (FP) was originally developed at PNNL and has been further refined through this program and has proven invaluable by allowing our domain scientists to program the high-level physics descriptions without having to deal with the low-level details of parallel processing. The preprocessor has been used to develop the parallel flow, reactive transport, and multiphase modules of the subsurface simulator. The preprocessor library, when used with the associated preprocessor, automatically distributes arrays, supports task and data parallelism, implements parallel I/O, and other translations required to use distributed memory, SMP, and clustered computing facilities at PNNL. It has been applied on both NWMPP1 and NWECS1 as it can explicitly support standard and SMP memory hierarchies. The resulting parallel code has been shown to be portable, efficient, and scalable.

A parallel real-time visualization capability (pV3) was implemented on this project to view simulation results as they are being calculated on the IBM SP. This approach eliminates the need to store data for post-processing which can often require hundreds of GB for a single simulation. It also allows us to correct errors in the simulation before more computational time is wasted. Our subsurface visualization capability was documented in a trade journal and is now the subject of a collaboration with IBM on their latest visualization hardware, the scalable graphics engine (SGE).

A library, D4, of parallel Krylov solvers was developed based on the message-passing paradigm and callable from FP programs. The D4 library implements and refines some state-of-the-art Krylov based methods for solving unsymmetric systems of linear equations with complex 
eigenvalues. The number of synchronization points in BiCGstab(L) \& ML(K)BiCGstab was significantly reduced by rearranging floating-point operations and grouping successive innerproducts together.

A new chemical reaction solver whose robustness far exceeds standard technologies was developed. This solver addresses a problem of long standing in the field of reactive transport: the poor convergence of the chemistry when transport has significantly altered the chemical conditions. With the new solver, we no longer have to repeat the entire transport and reaction calculations with a reduced timestep. This solver was the subject of an invited presentation at the SIAM Geosciences Conference (March 24-27, 1999).

A new dynamic load balancing algorithm was developed and implemented by the University of Texas to address imbalance in the chemistry workload across the processors. Dynamic balancing is necessary because the reaction fronts move through the domain with time. This low overhead algorithm obtained good scaling performance on 4 to 256 nodes of the PNNL SP on a problem dominated by chemistry work which was extremely localized (within two reaction fronts).

As indicated above, the project has been very successful developing the enabling technologies that the subsurface simulation capability is built on. This has provided a solid foundation for the testing and verification of the component models with the initial linkage of these components demonstrated on a hypothetical leak from the SX-109 tank. We continue work on the nonisothermal and density-dependent flow capabilites which are scheduled to be incorporated later this summer. The Java-based graphical interface is being updated to accommodate the additional capabilities. The project has been quite productive in publications/presentations and the PIs have been recognized in the subsurface simulation community through invited papers, session chairs, and positions on conference organizing committees.

The project team supported the SSI Expo by providing an animation sequence of a biogeochemical reactive transport simulation and images showing the SX-109 tank leak simulation with mineral dissolution and precipitation. The parallel Fortran Preprocessor and $\mathrm{pV} 3$ visualization capabilities were also submitted to the SSI Expo in the form of Powerpoint slides.

A fundamental scientific advancement supported by the GC4 grant is the refinement of the governing equations for multiphase fluid flow at the Darcy continuum scale. In the second year, LUDWIG, a lattice Boltzmann code developed at PNNL was ported to NWMPP1 to simulate subsurface fluid flow with multicomponent chemical and microbial kinetic reactions. LUDWIG was parallelized using the Global Array Tools library developed at PNNL. Initial grid meshes ranged from 1.0 to 3.4 million nodes. This work is being coordinated with high resolution mapping of a three-dimensional pore network using NMR imaging instrumentation at EMSL at PNNL.

A key difficulty of simulating subsurface processes is the need for bulk parameterizations to represent behaviors that integrate over multiple length scales of spatially variable material properties. PNNL is using NWMPP1 simulations of highly-resolved depictions of micro-scale subsurface environments to determine these effective parameters through a mechanistically 
based upscaling procedure. In this case, the diffusive transport of reactive solutes in biofilms was examined to determine the effective (upscaled) diffusivity in biofilm systems as a function of (i) microscale geometry, (ii) the microscale transport properties, and (iii) the protein-mediated transmembrane transport kinetics. The importance of this work is that it helps to explain what microscale factors are important for describing mass transfer in biofilms and tissues. This may have relevance for such diverse research areas as:

- engineering biofilms with specific properties for environmental remediation

- understanding how chemically-coded information is passed among cells in cellular systems

- predicting the rate of drug delivery to the cells of specific tissues

In addition to the inherent variability of the subsurface, the subsurface is also difficult to characterize because of the expense required to get reasonably detailed information. The availability of relatively few high quality samples of the subsurface equates to an inherent uncertainty in the problem specification and, as a result, uncertainty in the model predictions. PNNL is using maximum likelihood estimation for the uncertainty analysis of subsurface simulations. The purpose of maximum likelihood estimation in the context of linear models is to solve a system of over-determined linear equations $A x=b+e$ in a way that maximizes the likelihood of the response vector $b$ and to estimate its covariance. The covariance matrix itself plays a role in many other applications ranging from bio-informatics, econometrics to crop management. Parallel algorithms and code for restricted maximum likelihood covariance estimation using adaptive covariance REML techniques have been developed based on the PETSc Toolkit. Simulations with one million linear equations and 55 distribution parameters have been solved on NWMPP1.

The multifluid component of the GC4 project was applied to the modeling of radionuclide migration through the subsurface from leaked single-shell tanks is particularly challenging because of the strong thermal and chemical environments surrounding the tanks. Elevated tank temperatures requires consideration of soil drying below residual moisture conditions and recondensation of evaporated soil moisture; and the strong chemical environment, caused by the leaking nitrate and alluminate solutions, have the potential to alter the soil structure and binding characteristics for certain radionuclides. This component of the grand challenge group has been applying parallel implementations of multifluid reactive flow and transport software developed at the Pacific Northwest National Laboratory for investigating radionuclide migration issues at the SX Tank Farm on the Hanford Site. Of particular interest were the tanks surrounding the SX-108, SX-109, SX-111, and SX-112 tanks, all of which were used from 1956 to 1975 for storage of high-level waste from the Redox process at Hanford, and are known to have leaked. The primary focus for these investigations has been in modeling a cluster of six tanks, including the four listed above and the neighboring SX-105 and SX-106 tanks using computational grids of roughly one million nodes, providing a grid resolution of less than a cubic meter.

The uncertainty about waste behavior in the vadose zone has caused concern about SST waste retrieval strategies, which may involve hydraulic sluicing, and their impact on the vadose zone; assessment of health risks; and the validity of models that couple the Richards' and advectivedispersive equations to predict field-scale transport. Previous attempts to predict vadose zone transport have neglected driving forces and mechanisms of nonuniform contaminant migration, 
which result in erratic flow patterns and cause contaminants to bypass much of the unsaturated soil matrix. The overall research approach combines laboratory, field, and numerical experimentation. Theories developed from laboratory and field experimentation are being incorporated into the multifluid subsurface flow and reactive transport simulators developed at the PNNL. As with the other numerical tasks associated with the subsurface flow and reactive transport component code development is following a two stage process, where serial coding is first developed and verified in Fortran 90 and then converted into a parallel implementation through a Fortran Preprocessor developed by PNNL.

In the second year, building on previous developments of efficient strategies for dynamically balancing the chemistry workload in the reactive transport simulator ParSSim, the University of Texas studied the behavior of a strongly coupled flow/transport/reaction problem. The reactive infiltration instability is well-known: when a fluid dissolves all or part of a porous medium, the permeability of the medium increases, so that more fluid enters that region. Perturbations are therefore amplified, and the evolution of flow paths is of both theoretical and practical interest. It is also a computationally intensive application, because the flow field must be recalculated frequently. They have studied the frequency of such updates required for correct representation of the physics. This is nontrivial in heterogeneous domains (geostatistical initial distributions of permeability). Results for non-reactive transport illustrate the importance of hierarchical geological descriptions; the behavior in the reactive system has not yet been fully catalogued.

An interesting spinoff of this work has arisen. The flow computation in ParSSim makes heavy use of basic linear algebra operations, and the library for such operations on the SP has been highly optimized. Indeed, for the coupled problems described above, the computation is about six times faster on the SP than on the same number of Pentium II processors, even though the PII's have approximately three times faster clock speeds. This observation suggests a solution via heterogeneous computing: running the flow calculations on a platform such as the SP while running the transport and chemistry calculations on a local platform to facilitate output handling and visualization.

Also in the second year, PNNL developed and tested a multidimensional implementation of the Exact Linear Parts algorithm ( Beylkin, Keiser and Voizovoi, J. Comp. Phy. 1999) for regions with large variations. This is a multi-wavelet semigroup based algorithm. The multiwavelet approach has the advantage of localized support and multi-resolution adaptivity in spatial variations and higher order terms. For $\mathrm{M}$ time levels involved in the discretization, the explicit algorithm has order M accuracy. The explicit algorithm scales well on massively parallel computers.

In the third year of this project, three new activities were started: 1) parallelization of the reactive transport simulator, $\mathrm{CRUNCH}, 2$ ) computational fluid dynamics using NWGrid and NWPhys, and 3) calculation of effective diffusivity in cellular systems such as biofilms and tissues. Continuing activities from previous years included

- incorporating and testing PETSc linear solver routines in the multiphase fluid flow nonlinear iteration scheme, 
- increasing the efficiency in the. arbitrary covariance restricted maximum likelihood algorithm,

- enhancing the chemistry capabilities in the lattice Boltzmann modeling technology and applying it to a glass waste form, and

- reducing the numerical dispersion in long-term transport calculations through the use of characteristics-mixed methods as part of an Eulerian-Lagrangian localized adjoint method.

The parallel implementation of PNNL's STOMP simulator has been successfully applied to problems involving isothermal multifluid flow, but has demonstrated convergence problems with iterative linear system solvers for nonisothermal multifluid processes with strong coupling between the thermal and hydrologic fields. We took advantage of the high-level coding approach afforded by the parallel Fortran Preprocessor, which allows our source code to be compiled and executed without modification on any workstation with a FORTRAN-90 compiler. By comparing results from parallel and serial implementations of STOMP, we identified the root cause of numerical convergence problems that developed for highly coupled thermal and multifluid hydrologic transport. The Portable, Extensible Toolkit for Scientific Computation (PETSc) was incorporated into the STOMP simulator to investigate and implement solution techniques for strongly coupled nonisothermal multifluid porous media flow equations for a parallel computing environment. The developed scheme was tested on a series of field-scale problems involving a cluster of self-boiling and leaking underground storage tanks on the Hanford Site. Using parallel and serial implementations of STOMP90, these simulations were executed using the biconjugate gradient stabilized solver and incomplete LU factorization preconditioner with three levels of fill offered by SPLIB and PETSc, respectively.

The CRUNCH simulator is a joint LLNL-PNNL development that calculates one-, two-, or three-dimensional advective and diffusive transport with multicomponent chemical reactions. It can also simulate a batch reactor as well as a reaction path. A mixed system of equilibrium and kinetic reactions representing aqueous and surface complexation, ion exchange, precipitation and dissolution is solved. The code accommodates steady-state and transient, saturated and unsaturated flow fields on structured cell centered grids. Irregular boundaries are handled through the specification of inactive grid cells. The simulator combines two modeling approaches, operator splitting and global implicit methods in a FORTRAN-90 based multicomponent reactive transport code. Steve Yabusaki worked with the Waterways Experiment Station to implement the operator split transport scheme as well as compatibility with the Groundwater Modeling System (GMS), a user environment for modeling and analyzing subsurface data. Matt Rosing applied his preprocessing technology to the FORTRAN-90 source code to generate the parallel FORTRAN-90 with MPI. The initial parallelization targeted the operator splitting modeling option. In this case, the transport calculations used nearest-neighbor communications, which was handled through a data-parallel approach; and the chemical reactions were treated with a grid cell by grid cell task parallel approach. Testing on the migration of cesium-137 from leaking single-shelled Hanford waste tanks as a function of multicomponent ion exchange is ongoing.

Over long simulation times even a small amount of physical diffusion and dispersion can significantly affect reactive transport applications. Numerical dispersion can have just as much 
artificial effect and thereby destroy the relevance of simulation. Methods that conserve mass locally are prerequisite for accurate results over long times. The University of Texas developed an Eulerian-Lagrangian transport technique for their mixed finite element simulator PARSSIM, that minimizes numerical dispersion through the use of the Modified Method of Characteristics. and the ELLAM family of methods. Because the flow lines are followed explicitly, relatively large time steps can be taken with no CFL constraint. The technique transports entire volumes of fluid explicitly along streamtubes. More recently, Douglas and coworkers have successfully extended the technique to two-phase problems (Douglas et al. 2000).

The transport and reaction of chemical species are important features of groundwater flows. To this end, several modifications and improvements were made to the PNNL lattice Boltzmann simulator, LUDWIG. The gas chemical kinetics packages CHEMKIN and SURFACE CHEMKIN were added for gas phase reactions, whereas, the KEMOD program, developed by George Yeh at Penn. State, was added for liquid phase reactions and solids dissolution. This capability was demonstrated by simulating flow through a fractured glass canister. The parallel version of LUDWIG is based on the Global Arrays tools developed at PNNL. This year, simulations were performed with the lattice Boltzmann method on geometries ranging from $100 * * 3$ to $150^{* *} 3$ nodes. The program showed near linear speedup up to approximately 128 processors. This method solves the flow, energy and transport equations for fluid in complex porous medium geometries. The lattice Boltzmann method was used to model detailed reaction and transport in porous media, such as fractured glass canisters. The lattice Boltzmann has been shown to be a useful tool in simulating the detailed flow, chemical transport and reaction on the pore scale.

The performance of the Arbitrary Covariance Restricted maximum likelihood (ACR) software developed by Malard has been made more than an order of magnitude faster in FY2001. ACR now relies on quasi-Newton bound constrained optimization algorithms from the Toolkit for Advanced Optimization (TAO) developed at the Argonne National Laboratory. We demonstrated speed-ups on 256 processors of both an IBM SP (with Power II nodes) at PNNL and at Cray T3E at NERSC with over 50\% efficiency. This work is the first demonstration that large-scale statistical estimation, such as maximum likelihood and restricted maximum likelihood estimation, can be carried out on massively parallel multicomputers and will serve as a basis for work in subsurface flow inverse modeling. PNNL scientists are using the ACR software on the PNNL IBM SP to investigate the impact on the uncertainty of subsurface flow and transport models that rely on simplifying assumptions of steady-state, one-dimensional flow; homogeneous and isotropic transport medium properties; and unit hydraulic gradient or pistonflow displacement in the unsaturated zone. Comparisons between the two approaches include the probability distribution of peak dose, the relative importance of parameters, and the value of site-specific data in reducing uncertainty.

This year, several problems were solved in parallel on irregular and unstructured meshes using the NWGrid and NWPhys software developed by Harold Trease at PNNL. The scaling of a computational physics calculation was studied with NWPhys using the boundary fitted, structured mesh and partitioned for 16 processors. Two sets of runs were made on a cluster of 64 Linux workstations with dual 500MHz PIII and 512MB memory Dell PowerEdge1300 nodes, and the Giganet/Emulex cLAN network. The scaling of the absolute time and 
grind-time (time/cycle/element) is approximately linear mainly because of the (near) optimal partitioning of the data and work per processor. For a problem defined to have 10,000 elements per processor, the scaling is relatively constant as the problem size and number of processors grow. Several new Global Array sparse matrix communications functions were designed and implemented to support parallel computing in NWPhys. The new functions were patterned after the Connection Machine CMMSL runtime library functions, except for the binning function which is new. The functions include:

- Scatter with sum, product, max, min

- Scan with copy, sum, max, min

- Pack/Unpack

- Binning (General M x N mapping)

- Sorting

The previous work on biofilms was extended this past year to the calculation of the effective diffusivity of cellular systems such as biofilms and tissues. A finite difference model is used to predict the effective diffusivity of a cellular system on the basis of the subcellular-scale geometry and transport parameters. The effective diffusivity is predicted for a complex 3-dimensional structure that is based on laboratory observations of a biofilm, and these numerical predictions are compared with predictions from a simple analytical solution and with experimental data. The results indicate that under many practical circumstances, the simple analytical solution can be used to provide reasonable estimates of the effective diffusivity. To our knowledge, this is the first time a representation for diffusion in biofilms or tissues (or other cellular media) has been developed starting from the subcellular scale. The approach accounts for 1) intercellular diffusion and reaction, 2) chemical species transport across the cell wall via a specific proteinmediated process, and 3) extracellular diffusion. These processes were combined at the subcellular scale, and volume averaged over a representative volume of cellular material to obtain a macroscopic scale representation.

\section{Publications Resulting From This Work}

Chilakapati, A. and S.B. Yabusaki (1999). "Nonlinear reactions and nonuniform flows." Water Resources Research 35(8):2427-2438.

Chilakapati, A., S. Yabusaki, W. MacEvoy, and J. Szecsody. Groundwater flow, multicomponent transport and biogeochemistry: development and application of a coupled process model.

Journal of Contaminant Hydrology, Volume: 43, Issue: 3-4, May, 2000, pp. 303-325.

Chilakapati, A. A characteristic-conservative model for Darcian advection. Published in Advances in Water Resources, 22(6):597-609, 1999.

Chilakapati, A. Optimal design of a subsurface redox barrier. Published in AIChE Journal, 45(6):1342-1350, 1999. 
J. Douglas, Jr., C.S. Huang, and F. Pereira, The modified method of characteristics with adjusted advection, Numer. Math., 83 (1999), pp. 353-369.

J. Douglas, Jr., F. Pereira, and L.M. Yeh, A locally conservative Eulerian-Lagrangian numerical method and its application to nonlinear transport in porous media, Comput. Geosci., 4 (2000), pp. $\sim 1--40$.

MacEvoy, W., A. Chilakapati, and S. Yabusaki. A robust solver for mixed kinetic-equilibrium reaction systems in groundwater modeling. In preparation.

Malard, J. (2000) "Parallel Restricted Maximum Likelihood Estimation for Linear Models with a Dense Exogenous Matrix", International Workshop on Parallel matrix algorithms and applications, 18-20 August 2000, Neuchâtel, Switzerland. Accepted for presentation, proceedings will appear in Parallel Computing.

Malard, J. and G.I. Fann, and M.D. White, Performance of a Parallel \{BiCGstab(L)\} Implementation on Subsurface Flow Modeling, Proceedings of the Fifth Copper Mountain Conference on Iterative. Methods", April, 1998.

Malard, J. and M.D. White, An orthogonal projection based method for use in parallel subsurface flow modeling, in preparation.

Malard, Joel. Parallel Restricted Maximum Likelihood Estimation for Linear Models with a Dense Exogenous Matrix. Accepted for publication in Parallel Computing, 2001.

Palmer, B.J. and D.R. Rector. 2000. "Lattice Boltzmann Algorithm for Simulating Thermal Two-Phase Flow," Phys. Rev. E, Vol. 61, No. 5, pp. 5295-5306.

Palmer, B.J. and D.R. Rector. 2000. "Lattice Boltzmann Algorithm for Simulating Thermal Flow in Compressible Fluids," Journal of Computational Physics, Vol. 161, pp. 1-20.

Palmer, B.J. and D.R. Rector. 2000. "Lattice Boltzmann Algorithm for Simulating Thermal Flow in Compressible Fluids", accepted by Journal of Computational Physics.

Rector, D.R. and B.J. Palmer. 1999. "Simulation of Microscale Chemical Separation Processes Using the Lattice-Boltzmann Method", Proceedings of the Third. International Conference on Microreaction Technology, Frankfurt, Germany.

Rector, D.R., J.M. Cuta, and B.J. Palmer. 1998. "Lattice -Boltzmann Code Development for Mirco-Fluidic Systems",. Proceedings of the Second International Conference on Mircoreaction Technology, New Orleans, Louisiana.

Rosing, M. and S. Yabusaki. A Programmable Preprocessor for Parallelizing Fortran90. Refereed paper accepted for SuperComputing ‘99, November 1999, Portland, Oregon. 
Scheibe, T.D. and S.B. Yabusaki. 1998. "Scaling of Flow and Transport in Heterogeneous Groundwater Systems." Advances in Water Resources, 22(3).

White, M. D. and M. Oostrom. 1998. "Modeling surfactant-enhanced nonaqueous-phase liquid remediation of porous media." Soil Science,. 163(12):931-940.

White, M.D., M.D. Williams, and M. Oostrom. 1999. "Simulating Gas Entrapment, Mobilization and Air Dissolution for Fluctuating Water Table Conditions." In Proceedings of Nineteenth Annual American Geophysical Union Hydrology Days, Fort Collins, Colorado, August 16-20, 1999. Hydrology Days Publication, Atherton, California.

Wood, B.D., M. Quintard, and S. Whitaker. 2000. "Numerical calculation of biofilm diffusivities," In preparation for Biotechnology and Bioengineering, 2000.

Wood, B.D., M. Quintard, and S. Whitaker. Calculation of Effective Diffusivities for Biofilms and Tissues. In press, Biotechnology and Bioengineering, 2000.

Yabusaki, S.B. K.J. Cantrell, B. Sass, and C.I. Steefel. 2001. "Multicomponent Reactive Transport in an In Situ Zero-Valent Iron Cell." Environmental Science and Technology, 35(7); 1493-1503.

Yabusaki, S.B., C.I. Steefel, and B.D. Wood. 1998. "Multidimensional, Multispecies Reactive Transport in Nonuniform Velocity Fields: Code Verification using an Advective Reactive Streamtube Approach.” Journal of Contaminant Hydrology, 30:299-331.

\section{Presentations Resulting From This Work}

Altmann, N., J. Malard, A. Tuson, and A. Smaill. An evolutionary approach for improving the cache usage of Fortran codes, High Performance Computing 1999 Grand Challenges in Computer Simulation,. San Diego, CA, (ed.) Tentner, A., 291-7, 1999.

B.J. Palmer and D.R. Rector. 2000. Lattice Boltzmann Simulations of Thermal Two-Phase Flow. 2000 Conference on Discrete Models for Fluid Mechanics in Santa Fe, NM.

Bryant, S., C. Dawson, and H. van Duijn. "A Study of Transport of Heavy Metal Ions in Groundwater," presented at SIAM Conference on Mathematical and Computational Issues in the Geosciences, San Antonio, TX, 24-27 March 1999.

Bryant, S., J. Eaton, T. Arbogast, and M. Wheeler. "Efficient parallel computation strategies for reactive transport models," Invited presentation H52E-2, EOS Transactions, AGU 1998 Spring Meeting, vol. 79, no. 17, April 28, 1998 supplement.

Bryant, S. "Efficient Computation of Coupled Processes in Field-Scale Reactive Transport", to be presented at Environmental Molecular Sciences Symposia \& First EMSL User Meeting, Pacific Northwest National Laboratory, Richland, Washington July 21-24, 1999. 
Bryant, S. "Efficient Computation of Coupled Processes in Field-Scale Reactive Transport", presented at Society for Engineering Science Annual Meeting, Austin, Texas, Oct. 21-24, 1999.

Bryant, S. "Efficient strategies for large-scale remediation simulation," Invited Presentation at Environmental Solutions Program Annual Research Review, Austin, TX, April 1998.

Bryant, S. "Frontier Issues in Managing Oil and gas Production", presented at the Resource Recovery Workshop at the Institute for Mathematics and it Applications, Minneapolis, 913 Feb. 2000.

Bryant, S. "Some Mathematical Problems in Reactive Flow", presented at Mathematik poroeser Medien workshop, Mathematishes Forschungsinstitut Oberwolfach, 23-29 Jan. 2000.

Chilakapati, A. and S. Yabusaki. Nonlinear reactions and nonuniform flows. SIAM Geosciences, March 1999, San Antonio, Texas.

Chilakapati, A. Optimal design of a subsurface redox barrier. SIAM Geosciences, March 1999, San Antonio, Texas.

Eaton et al. "Efficient Parallel Computation of Spatially Heterogeneous Geochemical Reactive Transport," Computational Methods in Water Resources XII, Hersonisos, Crete, June 13-21, 1998.

Eaton, J. "Parallel Reservoir Simulation: Lessons Learned and New Ideas," Center for Subsurface Modeling 8th Annual Industrial Affiliates Meeting, Austin, Texas, Oct. 1998.

Joel Malard. "Parallel Restricted Maximum Likelihood Estimation for Linear Models with a Dense Exogenous Matrix", International Workshop on Parallel matrix algorithms and applications, 18-20 August 2000, Neuchâtel, Switzerland. Accepted for presentation, proceedings will appear in Parallel Computing.

Palmer, B.J. and D.R. Rector. 1998. "Lattice Boltzmann Algorithm for Simulating Thermal Flow in Compressible Fluids", presented at the 7th International Conference on the Discrete Simulation of Fluids, Oxford.

Rector, D.R., B. Palmer, J. Nieplocha, and J. Ju. 2001. "Building an Application Domain Specific Programming Framework for Computational Fluid Dynamics Calculations on Parallel Computers." Tenth SIAM Conference on Parallel Processing for Scientific Computing, March 12-14, 2001, Portsmouth, Virginia.

Rosing, M. and S.B. Yabusaki. "A Programmable Preprocessor for Parallelizing. Fortran90", SuperComputing 99, Portland, Oregon Nov 99. 
Wheeler, M. "Synthetic environments for modeling subsurface and surface flows," Invited presentation, SIAM Joint Conferences on Parallel Processing for Scientific Computing, and Mathematical and Computational Issues in the Geosciences, San Antonio, TX, 24-27 March 1999.

White, M.D. and M. Oostrom (2000). STOMP Workshop, Universität Stuttgart, Institut fur Wasserbau, May 30-June 1, 2000.

Yabusaki, S., M. White, A. Chilakapati, M. Rosing, and G.I. Fann. Design and application of a parallel subsurface multiphase reactive transport simulator. SIAM Geosciences, March 1999, San Antonio, Texas.

Yabusaki, S.B. (1998). "In Situ Chemical Treatment in a Funnel and Gate Treatment System." 2nd Annual Department of Defense Environmental Security Modeling and Simulation Conference. May 4-6, 1998, Alexandria, Virginia.

Yabusaki, S.B. (1998). "Modeling Transport and Reactions in Nonuniform Flows." Gordon Research Conference on Modeling of Flow in Permeable Media. August 2-7, 1998, Proctor, New Hampshire.

Yabusaki, S.B. (2000). "Multicomponent Reactive Transport in an In Situ Zero-Valent Iron Cell." Institute for Mathematics and its Application Workshop on the Remediation of Hazardous Materials, January 2000, Minneapolis, MN.

Yabusaki, S.B. (2000). "Multiphase Flow and Reactive Transport beneath a Leaking HLW Tank." American Chemical Society Annual Meeting, March 2000, San Francisco, CA.

\section{$\underline{\text { List of Significant Methods/Routines or Codes Developed }}$}

\section{$\underline{\text { Parallel Preprocessor }}$}

Preprocessor library was developed that is used for parallelizing Fortran90 programs. The library, when used with the associated preprocessor, automatically distributes arrays, supports task and data parallelism, implements parallel I/O, and other translations required. to use distributed memory, SMP, and clustered computing facilities at PNNL.

$\underline{\text { Parallel Visualization. }}$

We have developed parallel visualization applications that are based on third-party software (i.e., pV3) and algorithms that were created with the use of OpenGL libraries. pV3 is based on pvm across the network whereas sockets are used for the OpenGL applications. 


\section{$\underline{\text { Parallel Linear Solver }}$}

A library of Krylov solvers, D4, has been developed based on the message-passing paradigm and callable from FP programs. The D4 library implements and refines some state-of-the-art Krylov based methods for solving unsymmetric systems of linear equations with complex eigenvalues.

\section{$\underline{\text { Advection Scheme }}$}

An adaptive multi-wavelet semi-group method for solving convection-advection equations has been developed. The differential operator and solutions are represented in the multi-wavelet basis and thus are compatible with the multi-resolution solution space that multi-wavelet provides. Currently we have an 8 multi-wavelet code that is being tested as a plug-in for subgrid modeling. It handles both periodic and non-periodic boundary conditions.

\section{$\underline{\text { Reaction Solver }}$}

A robust reaction solver for the system of differential algebraic equations has been developed for the solution of mixed equilibrium and kinetic biogeochemical reactions. The algorithm is based on a logarithmic reformulation of the reaction system, damped Newton-Raphson iteration, and homotopy to increase the basin of attraction for convergence.

\section{Load Balancing}

Dynamic load balancing for chemistry computations: operator splitting technique gives rise to chemistry subproblem that must be solved independently at every grid block. The routine packs up subproblems on processors that have an excess of chemical work and sends them to processors with a relative lack of chemical work; it then gathers up the results and sends them back to the processors that "own" the grid cells at which the chemistry subproblem was posed.

\section{$\underline{\text { Pore Scale Simulators }}$}

Development of the parallel version of LUDWIG, which is a flow and transport computer code based on the lattice Boltzmann simulation method. This program simulates fluid flow through complex geometries, transport of energy and chemical species by convection and diffusion and bulk and surface chemical reactions. This simulation capability is fundamental to the development of new scaling theories for flow, transport, and biogeochemical reactions.

\section{$\underline{\text { Parallel Multiphase Simulators }}$}

IPARS and STOMP90 are two parallel 3D multiphase flow and transport simulators designed to address a variety of subsurface environments, including variably saturated, multiple-phased, and nonisothermal. STOMP90's target capabilities were guided by proposed or applied remediation activities at sites contaminated with volatile organic compounds and/or radioactive material. It can also address variably frozen soils. 


\section{$\underline{\text { Parallel Reactive Transport }}$}

ParSSim - parallel subsurface simulator. Handles single phase flow and transport with arbitrary number of phases (fluid and solid; only only one fluid may flow) and chemical species. Both equilibrium and rate-limited reactions are supported. New features include possibility to specify number of transport time steps to be taken before updating the flow field. This is useful because the CFL number can change dramatically in a coupled system, and some means of dynamically adjusting the frequency of flow field updates is required. On-the-fly processing of concentration fields has been implemented to permit output of effluent concentrations for easy comparison of large-scale features of the flow.

MCTRACKER and SMART — parallel reactive transport simulators developed for highresolution, three-dimensional simulations of biogeochemistry in complex subsurface systems. MCTRACKER has been applied to systems with multiscale physical and chemical heterogeneities requiring more than 4 million grid cells.

CRUNCH - parallel reactive transport simulator for biogeochemistry in complex subsurface systems. Global implicit and operator split options for the solution of transport and reactions. Uses the EQ3/6 thermodynamic database to construct reaction stoichiometry and implement reaction parameters.

Complex Grid Mesh Generation - NWGrid and NWPhys software to address arbitrarily general multidimensional grids with matching and non-matching interfaces. These grids are particularly effective on subsurface material property distributions involving geologically complex systems with faults and engineered structures.

Maximum Likelihood - An efficient Arbitrary Covariance Restricted maximum likelihood (ACR) algorithm for massively parallel computers was developed based on quasi-Newton bound constrained optimization algorithms. The software calculates arbitrary covariance using the principle of maximum likelihood to (1) identify model parameters such as hydraulic conductivities, porosities, and storativities in heterogeneous and anisotropic media, and (2) estimate uncertainty in predicted values. The Arbitrary Covariance Restricted maximum likelihood method relies on quasi-Newton bound constrained optimization algorithms from the Toolkit for Advanced Optimization (TAO) developed at the Argonne National Laboratory.

Effective Cellular Diffusivity - A scheme was developed to numerically calculate the effective diffusivity of cellular systems such as biofilms and tissues. A finite difference model is used to predict the effective diffusivity of a cellular system on the basis of the subcellular-scale geometry and transport parameters. 


\section{Appendix A - Full report of First Year Activities and Accomplishments}

\section{$\underline{\text { Transport Methods }}$}

We have implemented and are experimenting with the application of adaptive multi-wavelet semi-group methods for solving convection-advection equations in groundwater applications. The differential operator and solutions are represented in the multi-wavelet basis and thus are compatible with the multi-resolution solution space that multi-wavelet provides. We are also experimenting with adaptive load-balancing using Global Array Toolkit to simplify program development as well as random access patterns on MPPs as the block sizes of wavelet coefficients are dynamically changed as well as redistributing the workload due to changing number of chemical reactions.

\section{Linear Solvers}

We have implemented a library D4 of Krylov solvers based on the message-passing paradigm and callable from FP programs. The D4 library implements and refines some state-of-the-art Krylov based methods for solving unsymmetric systems of linear equations with complex eigenvalues. The number of synchronization points in BiCGstab(L) \& ML(K)BiCGstab was significantly reduced by rearranging floating-point operations and grouping successive innerproducts together.

We have implemented the matrix condition estimators of Kenney and Laub known as small statistical sample estimates and integrated this method into the STOMP90 code. Such componentwise condition estimates can help pin-point sources of numerical instability in the computational domain. This information can then be used to refine boundary conditions or reformulate the PDE that models the relevant physical flows and reactions. Published results for the small sample estimates of covariance are for matrices that are much smaller than those encountered in practical subsurface multi-phase flow simulations. Preliminary experiments using larger problem matrices are encouraging. We are also investigating variance reduction methods for use in this context.

We are developing scalable algorithms for computing the correlation between field data and simulation results. The field data points can be taken at any point during the simulation period. Storage is saved by computing the correlation across time and space at various scales in real time as opposed to dumping to file the whole history of the simulation variable of interest. These computations are based on coarsening and smoothing operators available in D4.

\section{$\underline{\text { Parallel Programming Tools }}$}

The bulk of the time was spent developing a preprocessor library. The library is part of a preprocessor that generates portable and efficient parallel Fortran-90 code and has been successfully used in the development of a variety of environmental transport simulators at PNNL. The tool provides the basic functionality of a traditional preprocessor where directives are embedded in a serial Fortran program and interpreted by the preprocessor to produce parallel Fortran code. The unique aspect of this work is that the user can make additions to, or modify, these directives. 
The ability to modify the directives has allowed scientists to develop a single program on a serial machine and then quickly port it to more powerful parallel machines without having to modify the source code. This has several advantages over standard parallelization techniques. First, hardware resources have been efficiently used by scientists; most algorithm development has been done on scalar or small parallel machines. Second, it has been simple to port software between various parallel machines; a different preprocessor library was used for each architecture. Third, it has been easier for scientists to parallelize their code because relatively few directives were required. Fourth, the long term viability of the developed code is ensured. The scientists have developed well over 60,000 lines of modeling code while the preprocessor library is around 3,000 lines long. As parallel hardware and software environments change we will only have to modify the preprocessor library and not the bulk of the code developed.

A parallel visualization package, $\mathrm{pV} 3$, was also incorporated into the modeling codes developed. This required slight modification to the above mentioned preprocessor library. A very simple parallel visualization package was also developed. This was done as a backup to the $\mathrm{pV} 3$ package, as we were having difficulties making $\mathrm{pV} 3$ work. Depending on future problems with $\mathrm{pV} 3$, the simple library we developed may be expanded.

\section{$\underline{\text { Reaction Solver }}$}

Solution of even simple reaction networks can suffer from poor convergence in the iterative nonlinear root finding algorithms. This can happen when the starting guesses for concentrations are too far from the solution vector (outside the radius of convergence) or the search algorithm is moving erroneously away from the solution. Many conditions can exacerbate these problems including cancellation error from subtraction operations, poor condition numbers for the jacobian matrices, and nonlinearity of the underlying equations. Typically, the only recourse to failure of the chemistry solution is a reduction in time step and the repetition of the solution methodology. This is very expensive not only from the standpoint of repeating time steps but also for the multiple time steps that will now be performed in place of the original time step. To avoid this situation, a new reaction solver was developed to explicitly improve the robustness of the chemistry solution. To avoid cancellation errors, the solver eliminates all subtractions by separating negative and positive entries into two separate matrices. A logarithmic transform is used on all concentrations and the system is reformulated with a second application of logarithms on the quotient of the mass conservation equations. The effect of the reformulation is to reduce the condition number of the jacobian and make it more linear which results in a larger basin of attraction for the Newton-Raphson iteration scheme. We use a simple damping algorithm to address those cases where the Newton method is overshooting to a point that it cannot recover from. If the damped Newton fails, a homotopy or continuation method is employed to restart the root-finding from a known solution that is slowly changed until it exactly represents the desired problem.

The robustness of this approach far exceeds standard solution technologies. Recently, a new technique was added that approximates the system of nonlinear reactions with a series of piecewise linear functions. Computationally cheap evaluations allow the solver to quickly identify a guess near the root. This allows the previously developed log reformulation, damped Newton, and homotopy techniques to begin from a point that is in its radius of convergence. A 
mathematical proof guarantees convergence if there is a single positive root to the system. This new development holds promise for increasing the speed and robustness of these computations on the parallel computer.

A GUI front end, written in Perl and Java, has been developed for input preparation, development of the reaction networks, problem/data consistency checks, on-line code generation/ execution, and graphical analysis of results. The interface allows users to input arbitrarily complex reactions that are automatically processed by MAPLE (symbolic mathematical software) into FORTRAN routines for the functional and Jacobian evaluations. These routines are then compiled and linked into the parallel simulator. This approach is the most flexible arrangement available as new reaction forms and rate laws can be accommodated while maintaining the use of analytical jacobians which have been demonstrated to have advantages in solution efficiency. Another benefit of this approach is there are no opportunities to make errors in the coding of the solvers since source code is automatically created by MAPLE.

\section{Load Balancing}

While providing efficiency and accuracy, operator splitting of the chemistry, flow and transport computations can also provide opportunities for severe degradation of parallel scaling performance due to localized chemistry "hot spots" in the problem domain. This imbalance of chemical workload between processors occurs when the updating of chemical reactions becomes a set of independent computations, the intensity of which can vary widely in both time and space. Dynamic balancing is necessary (as opposed to allocating the processors differently at startup) because the reaction fronts move through the domain with time. UT-Austin developed and implemented routines to quantify and forecast the chemical workload for each processor. This low overhead dynamic load balancing algorithm incurs no performance penalty on problems for which the chemistry workload is small compared to the flow and transport workload. Good scaling performance was obtained by PARSSIM on 4 to 256 nodes of the PNNL SP on a problem dominated by chemistry work which was extremely localized (within two reaction fronts).

\section{Scaling of Fundamental Fluid Behavior}

LANL/LLNL/ND continued to refine the continuum scale multiphase equations by identifying more clearly the mathematical description of the critical equations, terms, and parameters needed to accurately simulate multiphase flow. This data includes spatial and temporal tracking of phase location, pressure, velocity; interface location and area, common line location and length, interface curvature, disjoining pressures. The microscale lattice Boltzmann simulations have focused on flow in small, simplified and geometrically constrained pore networks to compare model and theoretical predictions. A considerable effort was directed at correctly incorporating the microscale information into the macroscale equations, including constitutive equations for conservation of mass, momentum, and energy for phases, interfaces, and common lines. These simulations required using 36 equations with 157 unknowns. The microscale and macroscale work has been presented at two conferences, published in 2 major papers on the continuum method, and one on the microscale work. Five papers are in process, including one invited review paper. 


\section{$\underline{\text { Multiphase Flow }}$}

The fundamental objective for the MSFC has been the development of software for high performance computing of multifluid, nonisothermal flow through porous media systems. These objectives are being achieved through a full conversion of the Laboratory's multifluid subsurface transport simulator capabilities to parallel form, through the development of new Fortran 90 coding coupled with parallel coding directives. The approach involves scripting new Fortran 90 coding based on the STOMP simulator, a proven, configuration controlled and documented multiphase subsurface flow and transport simulator, which was originally coded in Fortran 77. The resulting Fortran 90 coding is then verified on a single processor against the STOMP simulator. Following a successful verification sequence, directives are inserted into the coding that are interpreted by the parallel Fortran preprocessor (fp) discussed above. A parallel implementation of the Fortran 90 coding is then created by using fp to interpret the parallel directives and reconfigure the original Fortran 90 coding into a Message Passing Interface (MPI) version of the source coding. The source coding. can then be compiled and linked with MPI libraries to generate a parallel executable. In conjunction with this development has been, the development of a parallel linear system solver, D4 (described above), that has been specifically designed to address nonsymmetric linear systems that result from the application of the NewtonRaphson iteration technique to the solution of the multiphase flow equations for porous-media systems. This approach is significant, in that it allows scientists and engineers to develop parallel implementations of scalar coding without being overly burdened with the details associated with the MPI language and allows the source coding to be portable across machine platforms and manufactures.

To demonstrate these new numerical capabilities for a subsurface flow problem relevant to the DOE's Hanford Site a parallel implementation of the aqueous-phase with gas entrapment operational mode of the simulator was applied to model a hypothetical leak typical of those at the SX Tank Farm on the Hanford Site. The hydrologic conceptual model for the site, including the subsurface stratigraphy, was developed from well-log data collected near and on the SX Tank Farm Area, in support of the monitoring and characterization activities. The completed simulation involved 1 million nodes (i.e., 100x100x100 nodes) and was executed on 64 processors of the NWMPP1. Previous attempts at modeling leak scenarios at the SX Tank Farm were limited to two-dimensional computational systems. The results revealed the previously unobserved shedding of surface recharge water around the tank surfaces and the complex nature of the leaking plume in response to the recharge water and hetergeneous stratigraphy of the subsurface site. The simulation additionally demonstrated the viability of developing parallel code using a parallel programming preprocessor tool, which has allowed each member of the development team to remain focused within their realm of expertise: multifluid hydrology, computer science, and applied mathematics.

\section{$\underline{\text { Reactive Transport }}$}

The current focus of the simulator development and application effort is directed at the simulation of the behavior of reactive fluids that leaked from the HLW tank SX-109. The new reaction solver technology was developed and tested on serial workstations, incorporated into the parallel simulator and applied to the tank leak problem. Of particular interest is the impact of the caustic, 
high-ionic strength tank fluids on the sediments underlying the waste tank. As the high ionic strength, high $\mathrm{pH}$, and high aluminum solution migrates through the soil environment, the Alhydroxides/oxyhydroxides will be the dominant mineral assemblage likely to form as the $\mathrm{pH}$ drops due to mixing with the ambient Hanford geochemical environment. Depending on how rapidly the $\mathrm{pH}$ drops, metastable Al-phases other than gibbsite may form. At high $\mathrm{pH}$ these could be amorphous Al-hydroxide, pseudoboehmite, and bayerite. These will recrystallize to form gibbsite given enough time, but the rate at which this occurs can depend on both the temperature and the $\mathrm{pH}$. Another unknown involves the actual surface area available for Alhydroxide/oxyhydroxide nucleation and precipitation (other mineral surfaces, for example, may be able to nucleate gibbsite). If the solution is sufficiently supersaturated, polymeric Al-species can form homogeneously in solution and adsorb to other mineral surfaces. These precipitates may be amorphous rather than crystalline.

The precipitation and dissolution of Al-hydroxides/oxides will lead to an evolving sediment pore structure where the porosity and the reactive surface area are dynamic. Porosity changes affect the hydraulics and, consequently, the transport of later arriving chemical species. Conversely, changes in mineral surface area affect the reactivity of the solid matrix with the later arriving chemical species. In the extreme, precipitation may plug the soil pores, resulting in the redirection of the tank liquids along new flow paths where the process may be repeated. Another possibility is that as more of the high-pH, high-Al solution comes into contact with the newly precipitated minerals (through percolation and/or accumulation), these minerals may begin to redissolve. The following figure shows the tank leak with graphs that show the changes to the geochemical system directly beneath the tank leak.
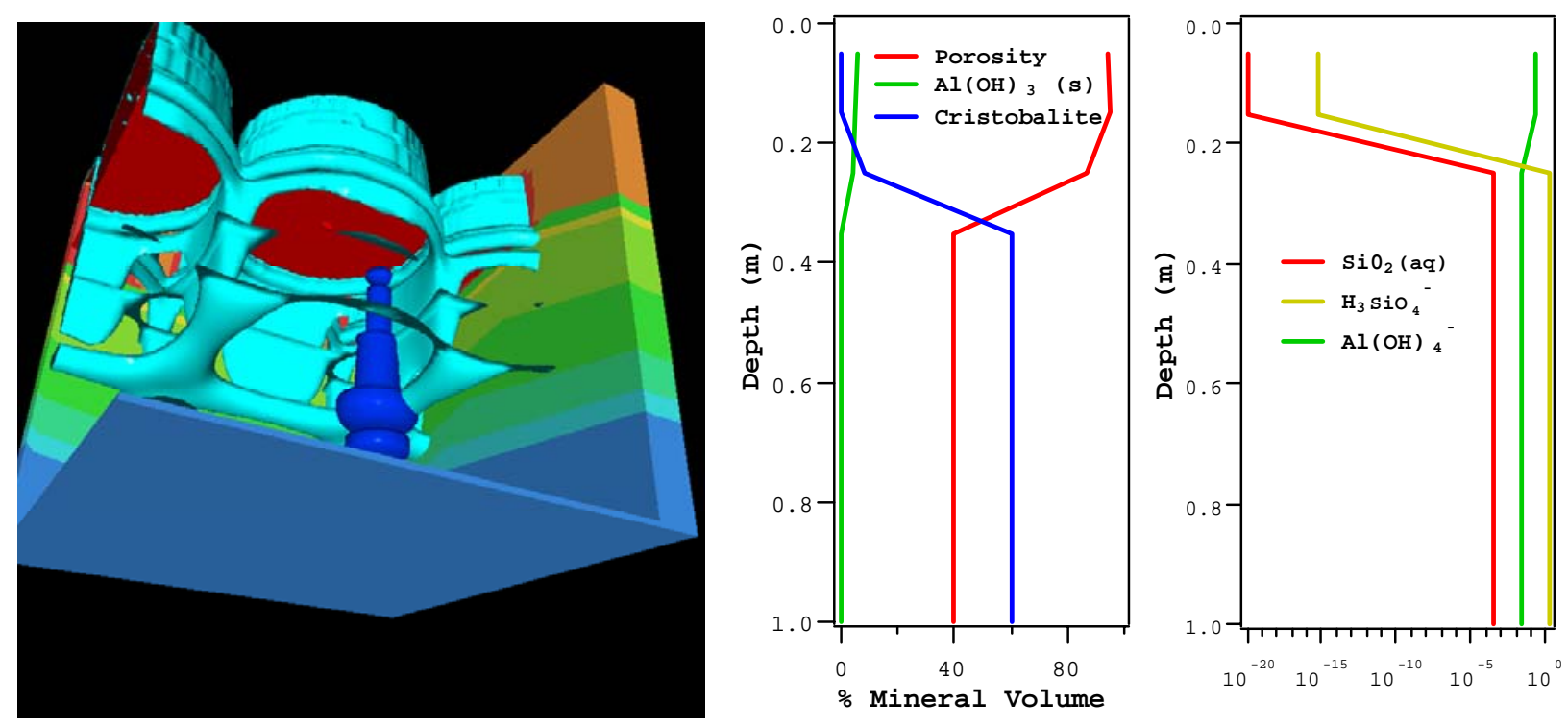

Figure 1. Simulated leak event beneath the SX Tank Farm.

In Figure 1, the light blue isosurface shows the increase in water content draped over the tanks in what is known as the "umbrella" effect. The dark blue isosurface is the hypothetical tank leak. The tanks are indicated in red and the stratigraphic sequence can be seen on the sides of the 
problem domain. The bottom boundary is the water table. In the graph on the left, cristobalite is being dissolved at the same time $\mathrm{Al}(\mathrm{OH})_{3}$ (s) is precipitating. On the right graph, the high dissolved silica concentration suppresses further cristobalite dissolution downstream, and aluminate is slowly being removed from the water column as $\mathrm{Al}(\mathrm{OH})_{3}(\mathrm{~s})$ precipitates.

There is an inherent load balancing issue when simulating a narrow leak of highly reactive fluid. Since most of the difficult chemistry is associated with the tank leak and the leak comprises a relatively small portion of the problem domain, the distribution of. computations is very unbalanced. This issue is currently being studied in the context of the parallel FORTRAN preprocessor. 


\section{Appendix B - Full Report of Second Year Activities and Accomplishments}

The goal of this project is to develop the first generation of models that fully address the coupling of dominant processes controlling the behavior of fluid, chemical and biological components in the subsurface. The large memory and computational performance of multiprocessor computing architectures will be exploited to provide modeling capabilities with unprecedented process detail and resolution to assess new scientific hypotheses, assist with experimental design, and to evaluate environmental technologies and remediation design. Moreover, the proposed capability developments will advance the scientific agenda for the subsurface through the realized advances in complex multiple-phase reaction modeling.

A fundamental scientific advancement supported by the GC4 grant is the refinement of the governing equations for multiphase fluid flow at the Darcy continuum scale. In the previous year, team members from LANL, LLNL, and the University of Notre Dame worked to identify the mathematical description of the critical equations, terms, and parameters needed to accurately simulate multiphase flow. At the microscale, small scale flow in explicitly modeled pores was modeled with a lattice Boltzmann technique. These simulations on simplified and geometrically constrained porous systems were used to refine the continuum theory. This past year LUDWIG, a lattice Boltzmann code developed at PNNL was ported to NWMPP1 to simulate subsurface fluid flow with multicomponent chemical and microbial kinetic reactions. LUDWIG was parallelized using the Global Array Tools library developed at PNNL. Initial grid meshes ranged from 1.0 to 3.4 million nodes. This work is being coordinated with high resolution mapping of a three-dimensional pore network using NMR imaging instrumentation at EMSL at PNNL.

A key difficulty of simulating subsurface processes is the need for bulk parameterizations to represent behaviors that integrate over multiple length scales of spatially variable material properties. PNNL is using NWMPP1 simulations of highly-resolved depictions of micro-scale subsurface environments to determine these effective parameters through a mechanistically based upscaling procedure. In this case, the diffusive transport of reactive solutes in biofilms was examined to determine the effective (upscaled) diffusivity in biofilm systems as a function of (i) microscale geometry, (ii) the microscale transport properties, and (iii) the protein-mediated transmembrane transport kinetics. The importance of this work is that it helps to explain what microscale factors are important for describing mass transfer in biofilms and tissues. This may have relevance for such diverse research areas as:

- engineering biofilms with specific properties for environmental remediation

- understanding how chemically-coded information is passed among cells in cellular systems

- predicting the rate of drug delivery to the cells of specific tissues

In addition to the inherent variability of the subsurface, the subsurface is also difficult to characterize because of the expense required to get reasonably detailed information. The availability of relatively few high quality samples of the subsurface equates to an inherent uncertainty in the problem specification and, as a result, uncertainty in the model predictions. PNNL is using maximum likelihood estimation for the uncertainty analysis of subsurface simulations. The purpose of maximum likelihood estimation in the context of linear models is to solve a system of over-determined linear equations $A x=b+e$ in a way that maximizes the 
likelihood of the response vector $b$ and to estimate its covariance. The covariance matrix itself plays a role in many other applications ranging from bio-informatics, econometrics to crop management. Parallel algorithms and code for restricted maximum likelihood covariance estimation using adaptive covariance REML techniques have been developed based on the PETSc Toolkit. Simulations with one million linear equations and 55 distribution parameters have been solved on NWMPP1.

An important technical outcome of the GC4 team is a coupled multiphase flow and reactive transport simulation capability. The sophistication of this approach is directly applicable to fundamental science as well as engineering needs at DOE cleanup sites. In the first year of the GC4 project, million-node isothermal simulations of leak behavior beneath a HLW tank at Hanford were performed to address the impact of highly resolved physical and chemical heterogeneities on subsurface flow, transport, and reactions. This year the four principal objectives for the multifluid component of the GC4 project were the application of developed scientific software to the 1) investigation of radionuclide migration in the deep vadose zone at the Hanford Site from leaking single-shell tanks; 2) simulation of clastic dike structures in the vadose zone at the Hanford Site; 3) investigation of unstable fingered flow in the unsaturated zone from infiltrating dense brines; and 4) prediction of DNAPL migration and remediation strategies for the Brooklawn Site, near Baton Rouge, Louisiana.

The modeling of radionuclide migration through the subsurface from leaked single-shell tanks is particularly challenging because of the strong thermal and chemical environments surrounding the tanks. Elevated tank temperatures requires consideration of soil drying below residual moisture conditions and recondensation of evaporated soil moisture; and the strong chemical environment, caused by the leaking nitrate and alluminate solutions, have the potential to alter the soil structure and binding characteristics for certain radionuclides. This component of the grand challenge group has been applying parallel implementations of multifluid reactive flow and transport software developed at the Pacific Northwest National Laboratory for investigating radionuclide migration issues at the SX Tank Farm on the Hanford Site. Of particular interest were the tanks surrounding the SX-108, SX-109, SX-111, and SX-112 tanks, all of which were used from 1956 to 1975 for storage of high-level waste from the Redox process at Hanford, and are known to have leaked. The primary focus for these investigations has been in modeling a cluster of six tanks, including the four listed above and the neighboring SX-105 and SX-106 tanks using computational grids of roughly one million nodes, providing a grid resolution of less than a cubic meter.

The uncertainty about waste behavior in the vadose zone has caused concern about SST waste retrieval strategies, which may involve hydraulic sluicing, and their impact on the vadose zone; assessment of health risks; and the validity of models that couple the Richards' and advectivedispersive equations to predict field-scale transport. Previous attempts to predict vadose zone transport have neglected driving forces and mechanisms of nonuniform contaminant migration, which result in erratic flow patterns and cause contaminants to bypass much of the unsaturated soil matrix. The primary objective of this ongoing EMSP research is to investigate the causes and extent of nonuniform flow in the vadose zone, and its effects on the migration of contaminants leaked from SSTs. This project will: (1) investigate the effect of elevated surface tension of highly saline fluids on wetting front instability, finger formation, and contaminant 
mobility; (2) investigate the conditions under which osmotically-driven vapor flux is operative and quantify its impact on plume transport, and (3) develop and incorporate theory describing these processes into an existing DOE-developed, numerical simulator to allow prediction of contaminant migration at realistic spatial and temporal scales. The overall research project combines laboratory, field, and numerical experimentation at the Pacific Northwest National Laboratory (PNNL), Oregon State University, and the Desert Research Institute. Theories developed from laboratory and field experimentation are being incorporated into the multifluid subsurface flow and reactive transport simulators developed at the PNNL. The fundamental numerical challenge associated with this research project involves creating efficient adaptive grid algorithms for parallel implementation across a variety of computing platforms. As with the other numerical tasks associated with the subsurface flow and reactive transport component code development is following a two stage process, where serial coding is first developed and verified in Fortran 90 and then converted into a parallel implementation through a Fortran Preprocessor developed by PNNL. The combination of conceptual, laboratory, and field experiments is providing the basis for understanding the driving forces and complex mechanisms affecting the migration of contaminants leaked from tanks. The result will be a computational tool that DOE can use to perform realistic analyses and predictions of contaminant transport in the vadose zone; evaluate different SST waste retrieval strategies and their impact on the vadose zone; and assess the health. Beyond the DOE complex, results will be useful in understanding the movement of accidentally or intentionally deposited saline fluids in the natural environment.

Clastic dikes at the Hanford Site may provide preferential pathways capable of enhanced vertical movement of moisture and contaminants through the vadose zone. Clastic dikes occur at many locations in both the 200 West and 200 East areas, and could explain deep migration of contaminants. However, reliable data on the dikes are scarce. This ongoing EMSP research project ${ }^{1}$ includes field and modeling studies on the spatial distribution of clastic dikes, the hydrologic properties within dikes, and the potential effect of clastic injection dikes on fluid flow through the vadose zone. The experimental components of this research are being conducted at an uncontaminated site adjacent to the S-SX Tank Farm, and will determine if clastic injection dikes provide fast paths for vertical transport through the vadose zone at Hanford. The associated numerical project to the primarily experimental classtic dike investigations is the ongoing laboratory-level LDRD project ${ }^{2}$ concerned with numerically modeling complex geometries in the subsurface. The principal thrust of this numerical project has been the development of capabilities for executing the recently developed multifluid subsurface flow and reactive transport models on complex grids to address preferential pathways, unstable flow structures, geologic discontinuities and engineered structures in the subsurface. The primary numerical challenge associated with this research is to develop numerical algorithms that allow computation on conforming grids while maintaining execution efficiencies on parallel platforms comparable to those for regular, structured grids.

The Brooklawn and Scenic Highway Sites, near Baton Rouge, Louisiana, are contaminated with chlorinated aromatic hydrocarbons and chlorinated hydrocarbons. Of particular interest is the

\footnotetext{
${ }^{1}$ Influence of Clastic Dikes on Vertical Migration of Contaminants in the Vadose Zone at Hanford,. EMSP 70193, Dr. Christopher J. Murray, Pacific Northwest National Laboratory

${ }^{2}$ Vadose Zone Modeling, PN99076/1404, Mark D. White and James A. Fort.
} 
hydraulic containment and source recovery activities within the batture area of the Brooklawn site, currently being implemented by NPC Services Inc., under a consent decree issued for site closure, which essentially involves pumping wells for the removal of organic liquids and contaminated groundwater. Numerical modeling, using the multifluid simulators developed by the Pacific Northwest National Laboratory, were applied to the Brooklawn Site to assess the value of the currently implemented hydraulic containment measures designed to control the migration of dense nonaqueous liquid (DNAPL) through the subsurface. Organics recovered from the pumping wells are incinerated and contaminated groundwater recovered from perimeter wells is treated on site before being pumped offsite toward the Mississippi River. Numerical investigations were conducted of DNAPL migration under different recovery and containment pumping scenarios to generate quantitative comparisons between hydraulic control options, providing a stronger scientific rationale for critical environmental management decisions. The Brooklawn site represents a significant computational challenge because of the numerous disposal sites, complex disposal histories, highly heterogeneous soil distributions, variable hydraulic conditions from fluctuations in the Mississippi River, and active pumping wells.

Several important supporting technologies were developed and/or refined in the first year of the project: (1) the parallel Fortran preprocessor (FP), (2) a parallel real-time visualization capability (pV3), (3) a library, D4, of parallel Krylov solvers was developed based on the message-passing paradigm and callable from FP programs, (4) a new chemical reaction solver whose robustness far exceeds standard technologies was developed, and (5) a new dynamic load balancing algorithm was developed and implemented by the University of Texas to address imbalance in the chemistry workload across the processors.

This past year, building on previous developments of efficient strategies for dynamically balancing the chemistry workload in the reactive transport simulator ParSSim, the University of Texas studied the behavior of a strongly coupled flow/transport/reaction problem. The reactive infiltration instability is well-known: when a fluid dissolves all or part of a porous medium, the permeability of the medium increases, so that more fluid enters that region. Perturbations are therefore amplified, and the evolution of flow paths is of both theoretical and practical interest. It is also a computationally intensive application, because the flow field must be recalculated frequently. They have studied the frequency of such updates required for correct representation of the physics. This is nontrivial in heterogeneous domains (geostatistical initial distributions of permeability). Results for non-reactive transport illustrate the importance of hierarchical geological descriptions; the behavior in the reactive system has not yet been fully catalogued.

An interesting spinoff of this work has arisen. The flow computation in ParSSim makes heavy use of basic linear algebra operations, and the library for such operations on the SP has been highly optimized. Indeed, for the coupled problems described above, the computation is about six times faster on the SP than on the same number of Pentium II processors, even though the PII's have approximately three times faster clock speeds. This observation suggests a solution via heterogeneous computing: running the flow calculations on a platform such as the SP while running the transport and chemistry calculations on a local platform to facilitate output handling and visualization. 
In the past year, PNNL developed and tested a multidimensional implementation of the Exact Linear Parts algorithm ( Beylkin, Keiser and Voizovoi, J. Comp Phy 1999) for regions with large variations. This is a multi-wavelet semigroup based algorithm. The multiwavelet approach has the advantage of localized support and multi-resolution adaptivity in spatial variations and higher order terms. For M time levels involved in the discretization, the explicit algorithm has order $\mathrm{M}$ accuracy. The explicit algorithm scales well on massively parallel computers. 


\section{Appendix C - Full Report for Third Year Activities and Accomplishments}

In the third year of this project, three new activities were started: 1) parallelization of the reactive transport simulator, CRUNCH, 2) computational fluid dynamics using NWGrid and NWPhys, and 3) calculation of effective diffusivity in cellular systems such as biofilms and tissues. Continuing activities from previous years include

- incorporating and testing PETSc linear solver routines in the multiphase fluid flow nonlinear iteration scheme,

- increasing the efficiency in the. arbitrary covariance restricted maximum likelihood algorithm,

- enhancing the chemistry capabilities in the lattice Boltzmann modeling technology and applying it to a glass waste form, and

- reducing the numerical dispersion in long-term transport calculations through the use of characteristics-mixed methods as part of an Eulerian-Lagrangian localized adjoint method.

Incorporation of PETSc Solvers in the STOMP Nonlinear Multiphase Fluid Flow Solution Scheme (PNNL: White, Consultant: Rosing)

The STOMP simulator is being used to investigate field-scale multifluid flow and reactive solute transport problems concerning the remediation and closure of underground storage tank type waste management areas on the Hanford Site. The parallel implementations have been successfully applied to problems involving isothermal multifluid flow, but have demonstrated convergence problems with iterative linear system solvers for nonisothermal multifluid processes with strong coupling between the thermal and hydrologic fields. We take advantage of the highlevel coding approach afforded by the parallel Fortran Preprocessor, which allows our source code to be compiled and executed without modification on any workstation with a FORTRAN90 compiler. By comparing results from parallel and serial implementations of STOMP, it has been possible to identify the root cause for the types of numerical convergence problems that develop for highly coupled thermal and multifluid hydrologic transport.

The Portable, Extensible Toolkit for Scientific Computation (PETSc) was incorporated into the STOMP simulator to investigate and implement solution techniques for strongly coupled nonisothermal multifluid porous media flow equations for a parallel computing environment. Of interest were the linearization and preconditioning of Jacobian matrices, with the objective of developing a solution scheme for parallel processing that exhibits convergence characteristic of sequential coding, and execution speed characteristic of parallel coding. The developed scheme was tested on a series of field-scale problems involving a cluster of self-boiling and leaking underground storage tanks on the Hanford Site. These types of simulations are currently being executed on two-dimensional grids using sequential processing to answer questions about the past and future migration of long-lived radionuclides that have leaked from the tanks. Sloping strata beneath the Hanford Site tank farms and tank specific thermal histories, make the nonisothermal fluid flow and reactive transport problem three-dimensional domain. 
This study used numerical simulation to investigate the coupled processes of multifluid flow, heat transfer, and solute transport in a heterogeneous geologic environment surrounding the subject row of single-shell tanks. Using STOMP and the serial implementation of STOMP90 these simulations were executed using the biconjugate gradient stabilized solver and incomplete LU factorization preconditioner with three levels of fill offered by SPLIB and PETSc, respectively. The parallel implementation of STOMP90 using the biconjugate gradient stabilized solver and incomplete LU factorization with zero levels of fill, however, failed to achieve converged solutions beyond the point in time where the thermal and hydrologic systems become strongly coupled (i.e., high thermal loading with strong evaporative loss). Convergence failures were due strictly to the lack of fill during preconditioning of the Jacobian matrix. Although phase transitions and primary variable switching delays convergence and without constraints can yield unstable phase flopping conditions, Newton-Raphson linearization with numerical derivatives and primary variable switching has proven to be robust and convergent for direct linear system solvers. This scheme functioned equally well for iterative linear system solvers with adequate preconditioning (e.g., incomplete LU with three levels of fill), but failed for the simpler preconditioning schemes (e.g., diagonal preconditioning, incomplete LU with no fill).

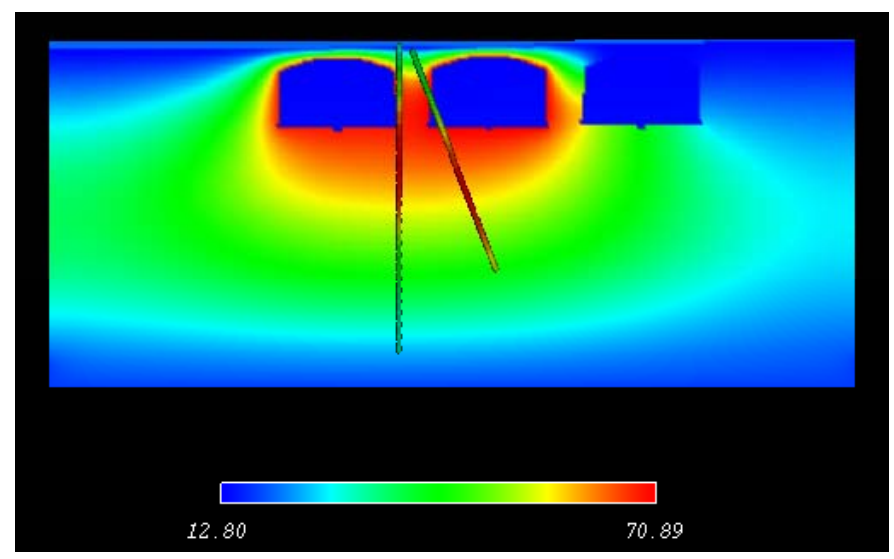

Figure 1. Predicted temperature distribution based on operational histories of tanks in the Hanford SX Tank Farm.

\section{Parallelization of the Reactive Transport Simulator, CRUNCH. (LLNL: Steefel, PNNL: Yabusaki, Consultant: Rosing)}

The CRUNCH simulator is a joint LLNLPNNL development. Carl Steefel combined two modeling approaches, operator splitting and global implicit methods in a FORTRAN-90 based multicomponent reactive transport code. Steve Yabusaki worked with the Waterways Experiment Station to implement the operator split transport scheme as well as compatibility with the Groundwater Modeling System (GMS), a

user environment for modeling and analyzing subsurface data. Matt Rosing applied his preprocessing technology to the FORTRAN-90 source code to generate the parallel FORTRAN90 with MPI.

CRUNCH calculates one-, two-, or three-dimensional advective and diffusive transport with multicomponent chemical reactions. It can also simulate a batch reactor as well as a reaction path. A mixed system of equilibrium and kinetic reactions representing aqueous and surface complexation, ion exchange, precipitation and dissolution is solved. An extended Debye-Huckel model is used to calculate activity corrections. Data from the EQ3/6 database is used to generate reaction stoichiometry and temperature-dependent thermodynamics from a list of user-provided primary and secondary species. Ionic radii for the activity model and specific mineral volumes are also included in the database. The code accommodates steady-state and transient, saturated 
and unsaturated flow fields on structured cell centered grids. Irregular boundaries are handled through the specification of inactive grid cells.

The initial parallelization targeted the operator splitting modeling option. In this case, the transport calculations use nearest-neighbor communications, which is handled through a dataparallel approach; and the chemical reactions are treated with a grid cell by grid cell task parallel approach. Testing on the migration of cesium-137 from leaking single-shelled Hanford waste tanks as a function of multicomponent ion exchange is ongoing.

\section{Accurate Transport Schemes for Long-Term Predictions (University of Texas - Austin)}

Due to inherent uncertainty in site characterization and physical process modeling, computer simulation can be indispensable in evaluating and tuning various groundwater contamination and remediation scenarios and risk factors. Such applications, however, present great challenges for meaningful simulation. For example, performance assessment of nuclear waste repositories requires simulation of flow, transport, dispersion, and nuclear and chemical reactions over extraordinarily long time periods to extraordinary accuracy_-part per trillion for some highly toxic species.

Over long times even a small amount of physical diffusion and dispersion can significantly affect reactive transport applications. Numerical dispersion can have just as much artificial effect and thereby destroy the relevance of simulation. Methods that conserve mass locally are prerequisite for accurate results over long times. Reaction rates depend on concentrations, which require both accurate masses and volumes, so it is just as important to conserves volume locally.

Among explicit methods, the Godunov method (LeVeque 1992) has the least numerical dispersion, and it uses a fixed grid, so volume is locally conserved, as is mass. However this method is subject to severe CFL time-step constraints, requiring many time steps over long time periods. Significant numerical dispersion is thereby introduced into the solution. Implicit methods can use longer time steps, but they produce even more numerical dispersion.

The Eulerian-Lagrangian techniques, such as the Modified Method of Characteristics (Douglas and Russell 1982), Douglas et al. 1999\} and the ELLAM family of methods (Celia et al. 1990), simulate transport in a Lagrangian coordinate system that follows the flow trajectories, and the rest of the physical phenomena in a simpler fixed Eulerian coordinate system. Because the flow lines are followed explicitly, relatively large time steps can be taken with no CFL constraint. If implemented correctly, numerical dispersion is negligible.

Arbogast and Wheeler (Arbogast and Wheeler 1995, Arbogast et al. 1992) have developed a characteristics-mixed method of ELLAM type that transports entire volumes of fluid explicitly along streamtubes. More recently, Douglas and coworkers have successfully extended the technique to two-phase problems (Douglas et al. 2000).

We illustrate the importance of reducing numerical dispersion with simulations of the far-field behavior of a leaking radioactive waste repository, devised from the Couplex1 test problem (http://www.andra.fr/fr/actu/archi-0030.htm). This problem is one of several designed to 
evaluate the capabilities of reactive transport simulators worldwide, and CSM has been awarded a small stipend to participate in a second round of testing and evaluation. The problem sets a leak rate for radioactive iodine, I129, and steady flow rates through limestone layers above and below a thick clay layer in which the repository is situated. The extremely low permeability of the clay results in flow velocities four to six orders of magnitude smaller in the clay than in the limestone. Dispersion is consequently much higher in the limestone as well, while molecular diffusion is a significant transport mechanism in the clay. Initial concentrations in the vicinity of the repository are of order unity, and we must track concentrations down to parts per trillion over a period of 10 million years. Figure 2 and Figure 3 show I129 plumes after 0.7 million years. Note that the CMM simulator has a much sharper appearance, as the Godunov method experiences a much greater level of numerical dispersion, obscuring the result. Moreover, the long time advance of the plume is improperly enhanced by the numerical dispersion in the later case.

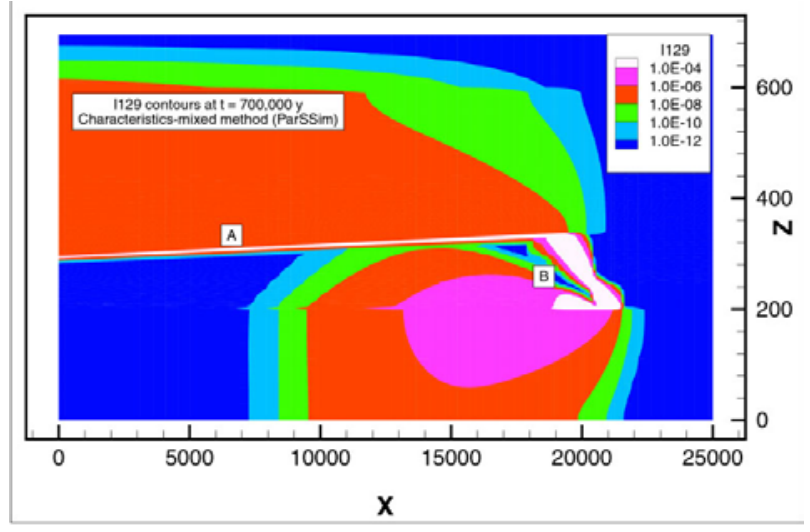

Figure 2. Simulation of 0.7 million years of leakage from a radioactive waste repository. The Characteristics-Mixed Method (CMM) captures important finescale features in a plume of Iodine-129 such as (A) the streak of high concentrations at the upper boundary of the clay layer and (B) the large gradients in concentration to the left of the repository.

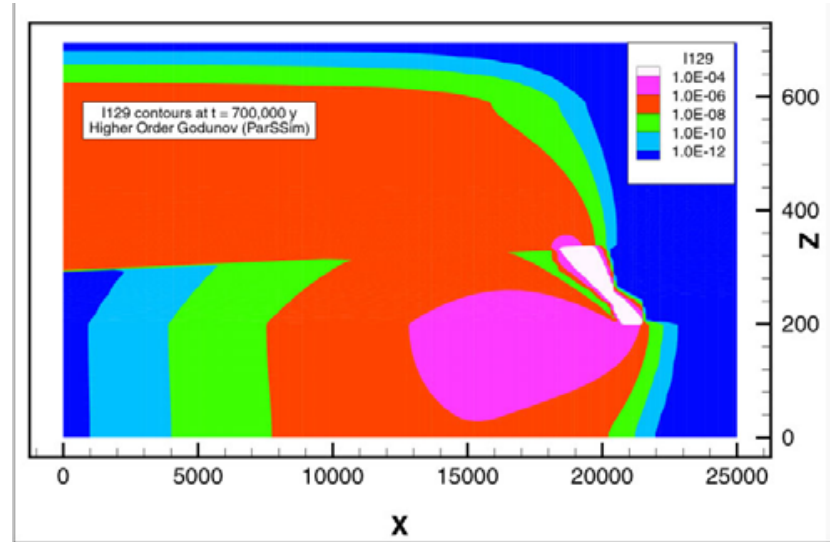

Figure 3. Same problem as in Figure 2 but simulated using a higher-order Godunov scheme. Numerical dispersion has obscured the features noted in Figure 2. Moreover the simulation predicts significantly faster advance of the contaminant plume in the limestone layer below the repository.

\section{Lattice Boltzmann Methods (DR Rector and BJ Palmer, PNNL)}

The macroscopic behavior of flow and transport in porous systems is determined by the physics of processes that occur on microscopic time and length scales. The lattice Boltzmann method allows the detailed simulation of complex geometries so that the macroscopic properties used in field scale simulations can be related to the microstructure of the porous medium. A major advantage of the lattice Boltzmann method over other CFD methods is that each node depends only on information from nearest neighbor nodes and does not require a global pressure array solution. 
The energy equation, including convection, conduction and latent heat of phase change, was implemented in the PNNL lattice Boltzmann simulator, LUDWIG. This allows the discrete modeling of multiphase systems including bubbles and droplets. Surface adsorption and wettability terms were included. This capability has been demonstrated by the simulation of bubble growth and lift off from a notched horizontal surface.

The transport and reaction of chemical species are important features of groundwater flows. To this end, several modifications and improvements were made to the PNNL lattice Boltzmann simulator. Chemical species transport equations were added to the lattice Boltzmann program. The gas chemical kinetics packages CHEMKIN and SURFACE CHEMKIN were added for gas phase reactions. The KEMOD program, developed by George Yeh at Penn. State, was added for liquid phase reactions and solids dissolution. This capability was demonstrated by simulating flow through a fractured glass canister.

A parallel version of LUDWIG using the Global Arrays tools was developed at PNNL. This year, simulations were performed with the lattice Boltzmann method on geometries ranging from $100 * * 3$ to $150 * * 3$ nodes. The program showed near linear speedup up to approximately 128 processors. This method solves the flow, energy and transport equations for fluid in complex porous medium geometries. The lattice Boltzmann method was used to model detailed reaction and transport in porous media, such as fractured glass canisters. The lattice Boltzmann has been shown to be a useful tool in simulating the detailed flow, chemical transport and reaction on the pore scale.

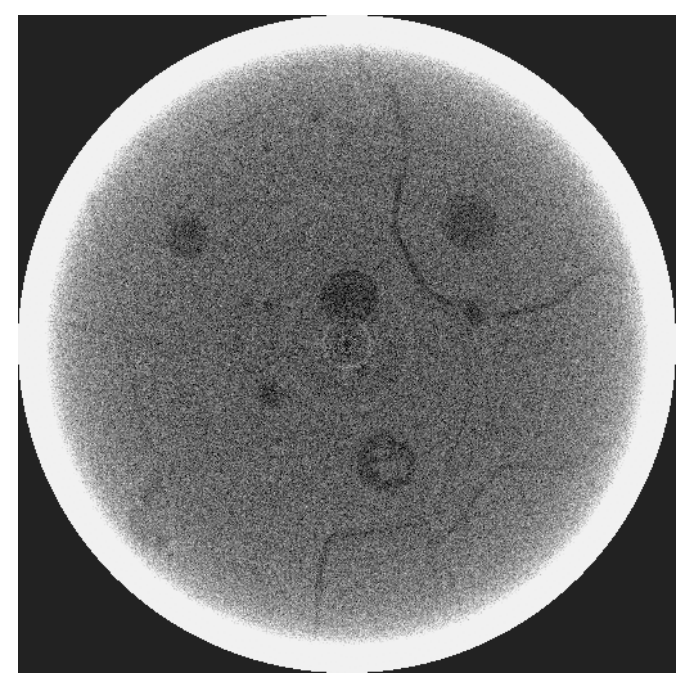

Figure 4. X-ray microtomography image for fractured HAN28 glass canister.

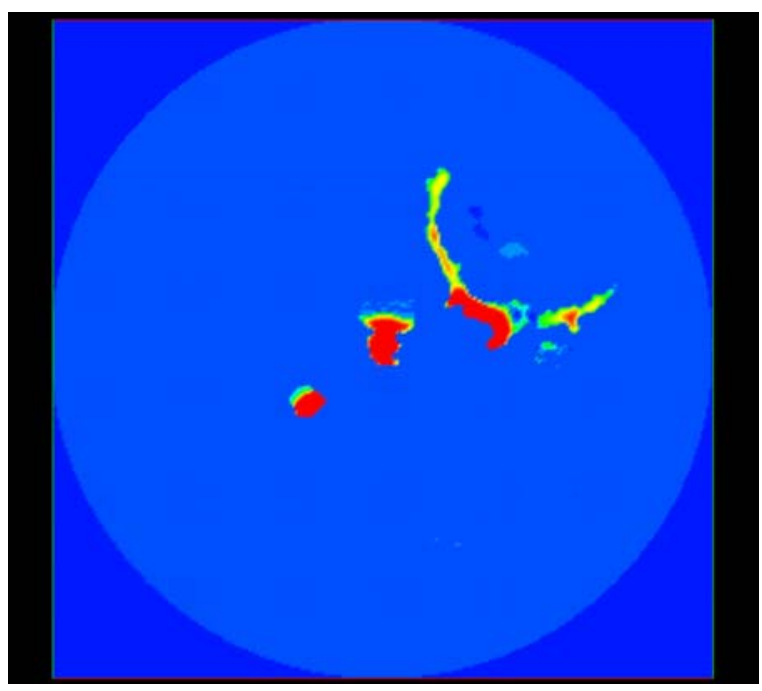

Figure 5. Predicted relative axial velocity for the same cross section as in Figure 1.

\section{Arbitrary Covariance Restricted Maximum Likelihood (ACR) (Malard, PNNL)}

Subsurface flow modeling of contaminated sites is a critical activity for the DOE. Computational models of subsurface flows delineate the current understanding of the physical laws affecting flow and transport of contaminants in the subsurface, and the process of 
environmental remediation. These models depend on parameters that cannot be measured directly over the entire simulation domain and period. The relevant field data is typically sparse, instantaneous and often irregularly spaced to accommodate regulatory objectives and logistic constraints. The potential impact on long term planning of computational models of contamination fate and transport prompts the importance of calibration and uncertainty analyses of these models. Of the several approaches used in this context, Carrera and Neuman have suggested using the principle of Maximum Likelihood to both identify model parameters such as hydraulic conductivities, porosities, and storativities in heterogeneous and anisotropic media, and estimate uncertainty in predicted values. The use of covariance matrix estimation also plays an important role in other application areas ranging from bio-informatics, econometrics to crop management. The performance of the Arbitrary Covariance Restricted maximum likelihood (ACR) software developed by Malard has been made more than an order of magnitude faster in FY2001. ACR now relies on quasi-Newton bound constrained optimization algorithms from the Toolkit for Advanced Optimization (TAO) developed at the Argonne National Laboratory. We demonstrated speed-ups on 256 processors of both an IBM SP (with Power II nodes) at PNNL and at Cray T3E at NERSC with over 50\% efficiency, see Figure. This work is the first demonstration that large-scale statistical estimation, such as maximum likelihood and restricted maximum likelihood estimation, can be carried out on massively parallel multicomputers and will serve as a basis for joint work with Shlomo Orr in subsurface flow inverse modeling.

Shlomo Orr, Phil Meyer and Joel Malard are using the PNNL IBM SP to investigate the impact on the uncertainty of subsurface flow and transport models of such factors as steady-state, onedimensional flow; homogeneous and isotropic transport medium properties; and unit hydraulic gradient or piston-flow displacement in the unsaturated zone. Because of their relative computational speed, such simplified models are particularly attractive when the impact of uncertainty in flow and transport needs to be evaluated. Simplifications in the representation of

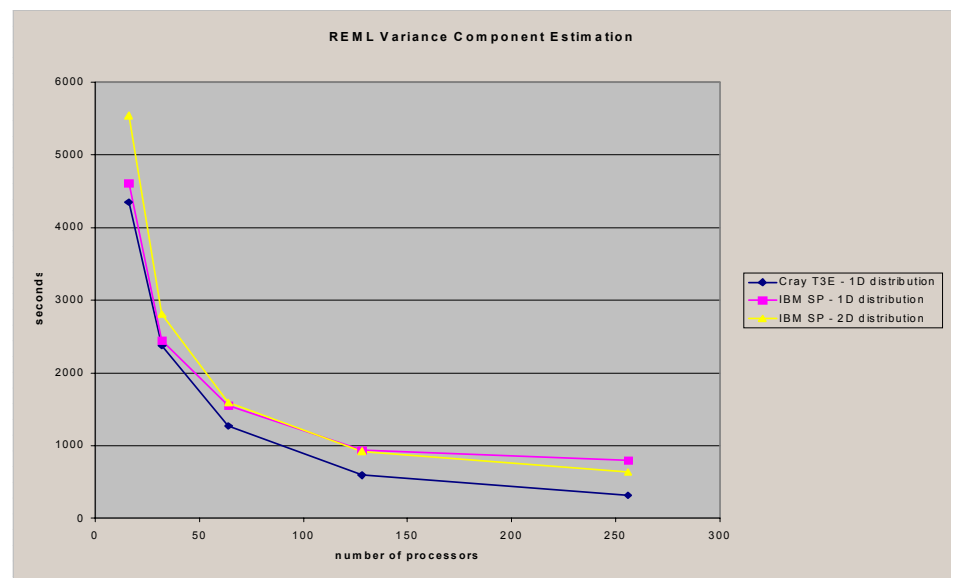

Figure 6. Parallel REML Estimation, 1,000,000 equations and 55 distribution parameters, to appear in Parallel Computing Journal, 2002 flow and transport can result in inaccurate estimates of the uncertainty in dose/risk, and may have important consequences for decisions based on the dose/risk assessments. Subsurface properties are derived from data obtained in the Las Cruces Trench experiments with source term data reflecting an actual decommissioning case. Comparisons between the two approaches include the probability distribution of peak dose, the relative importance of parameters, and the value of site-specific data in reducing uncertainty. 


\section{Physics and Chemistry on Irregular and Unstructured Grids (Trease, PNNL)}

The following are three problems being solved in parallel on irregular and unstructured meshes using NWGrid and NWPhys.

\section{Parallel Benchmarking and Optimization of NWGrid and NWPhys}

The scaling of a computational physics calculation was studied with NWPhys using the boundary fitted, structured mesh shown in Figure 7 and partitioned (for 16 processors) as shown in Figure 8. Two sets of runs were made on a cluster of 64 Linux workstations with dual $500 \mathrm{MHz}$ PIII and 512MB memory Dell PowerEdge-1300 nodes, and the Giganet/Emulex cLAN network. The first was a fixed size problem run with different numbers of processors. The second was a problem the grew in size as the number of processors increased. The scaling of the absolute time and grind-time (time/cycle/element) is approximately linear mainly because of the (near) optimal partitioning of the data and work per processor. For a problem defined to have 10,000 elements per processor, the scaling is relatively constant as the problem size and number of processors grow. The performance results are encouraging, especially since our initial emphasis was on providing functionality of these sparse matrix operations and not much time on tuning the performance of the tools. The optimal partitioning plays a big role in these performance results, but the efficiency of the communication and sparse matrix modules are also reflected.

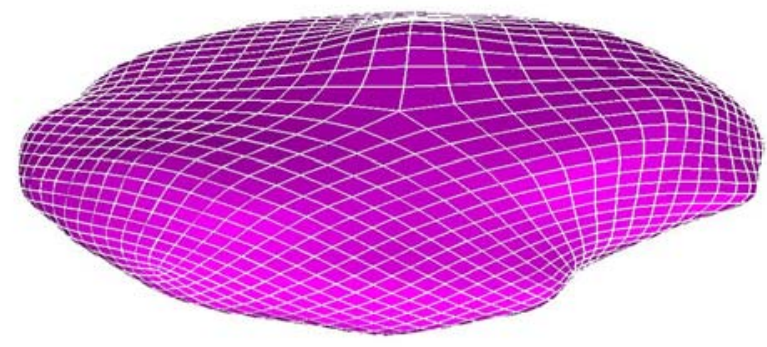

Figure 7. The a boundary fitted, unstructured mesh generated using NWGrid.

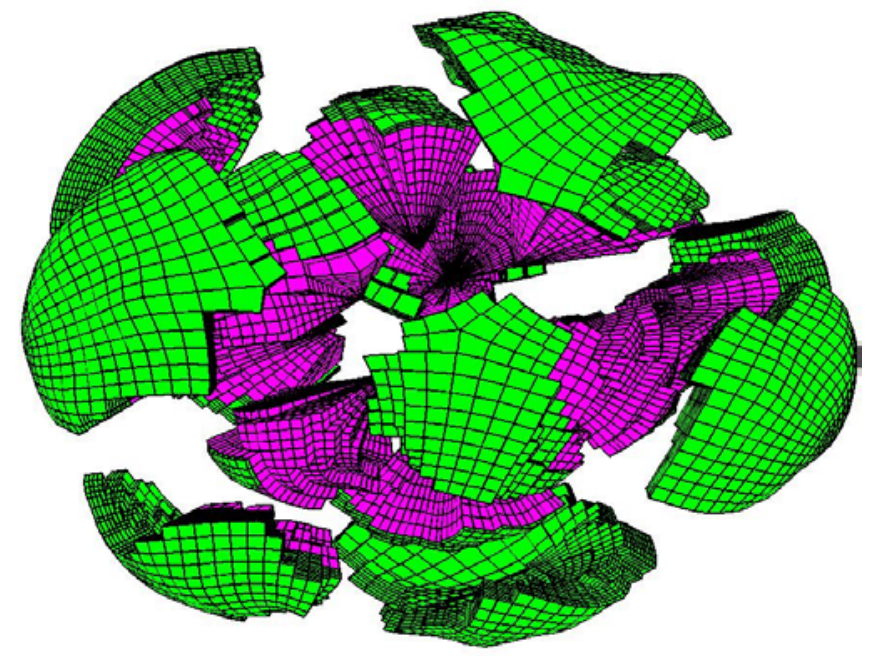

Figure 8. The 16-process version of an unstructured mesh used for benchmarking NWGrid and NWPhys.

\section{New Sparse Matrix Routines for Global Arrays}

Several new Global Array sparse matrix communications functions were designed and implemented to support parallel computing in NWPhys. The new functions were patterned after the Connection Machine CMMSL runtime library functions, except for the binning function which is new. The functions include: 
- $\quad$ Scatter with sum, product, max, min

- Scan with copy, sum, max, min

- Pack/Unpack

- $\quad$ Binning (General M x N mapping)

- Sorting

\section{Solving a Six Degree of Freedom Reaction Diffusion System on an Unstructured Mesh.}

Solving a six degree of freedom, time-dependent, reaction-diffusion equation system the concentration fields of reaction chemistry problem. Figure 1 show the 3-D mesh. Figure 2 shows the initial distribution for the $1^{\text {st }}$ field. The other 5 fields were initialized similarly. Figures 3-8 show the six steady state concentration fields. The problem was solved in parallel on Colony.
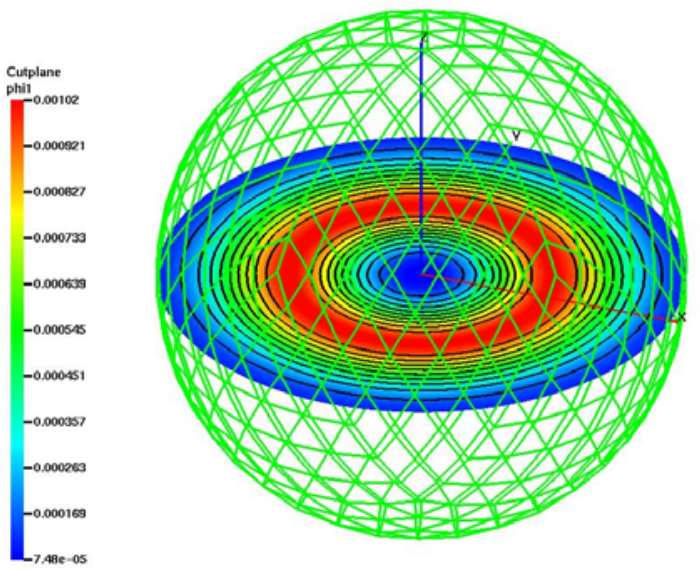

Figure 1. Initial grid and contour slice through the equator of the unstructured mesh show the $1^{\text {st }}$ concentration field.

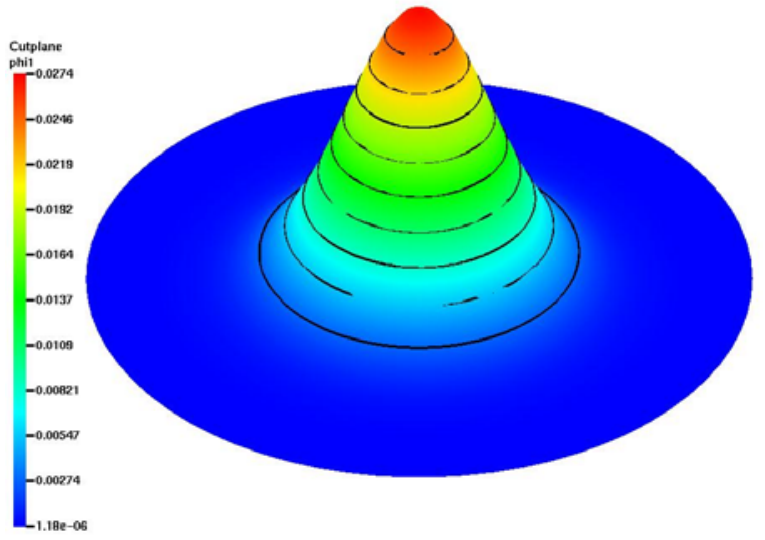

Figure 2. Equatorial slice of the $1^{\text {st }}$ concentration field at time $=0$. Here the height of the field is shown.
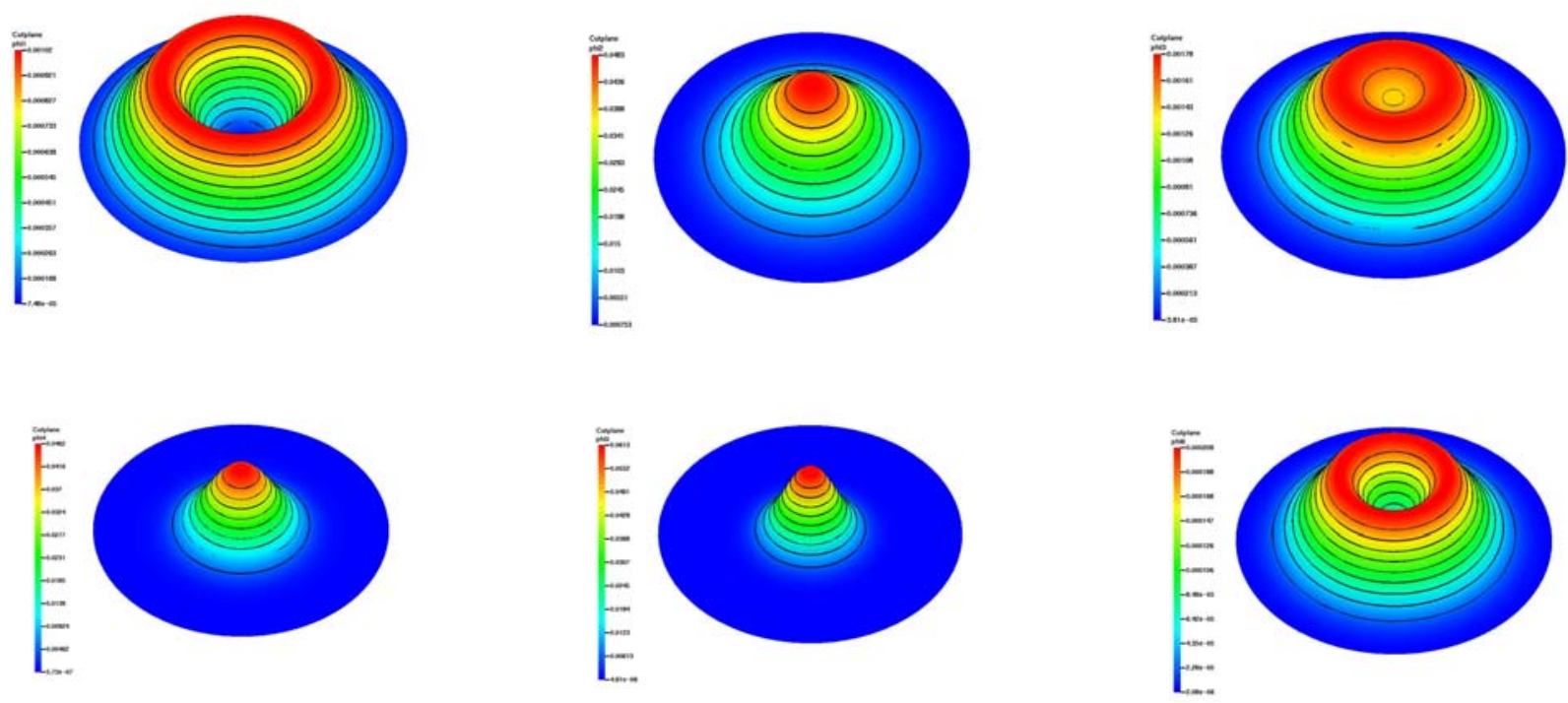

Figures 3-8. Equatorial slice of the concentration fields 1-6 at steady state, where height show the magnitude of the field. 


\section{Calculation of Effective Diffusivities for Biofilms and Tissues (Brian Wood, PNNL)}

In this work, a scheme was developed to numerically calculate the effective diffusivity of cellular systems such as biofilms and tissues. A finite difference model is used to predict the effective diffusivity of a cellular system on the basis of the subcellular-scale geometry and transport parameters. The effective diffusivity is predicted for a complex 3-dimensional structure that is based on laboratory observations of a biofilm, and these numerical predictions are compared with predictions from a simple analytical solution and with experimental data. The results indicate that under many practical circumstances, the simple analytical solution can be used to provide reasonable estimates of the effective diffusivity. To our knowledge, this is the first time a representation for diffusion in biofilms or tissues (or other cellular media) has been developed starting from the subcellular scale. The approach accounts for 1) intercellular diffusion and reaction, 2) chemical species transport across the cell wall via a specific proteinmediated process, and 3) extracellular diffusion. These processes were combined at the subcellular scale, and volume averaged over a representative volume of cellular material to obtain a macroscopic scale representation.

Computationally, the main challenge here was to determine the solution to the so-called closure problem that allowed us to predict the effective diffusion tensor on the basis of the subcellular scale geometry and transport parameters. The closure problem is predicted by a set of PDE's themselves (much like for problems in turbulence theory). For complex, 3-dimensional media, this calculation required significant computational resources. Additionally, we computed solutions over unit cells for a range of parameters, and to complete the calculations in a reasonable amount of time, it was necessary to have individual realizations of the system being computed simultaneously on multiple processors.

The results of this study have provided some important results. One of these results is that the work has helped to bound the conditions for which a simple analytical solution will provide reasonable estimates for the effective diffusion tensor, and when the more complicated numerical approach must be taken. 


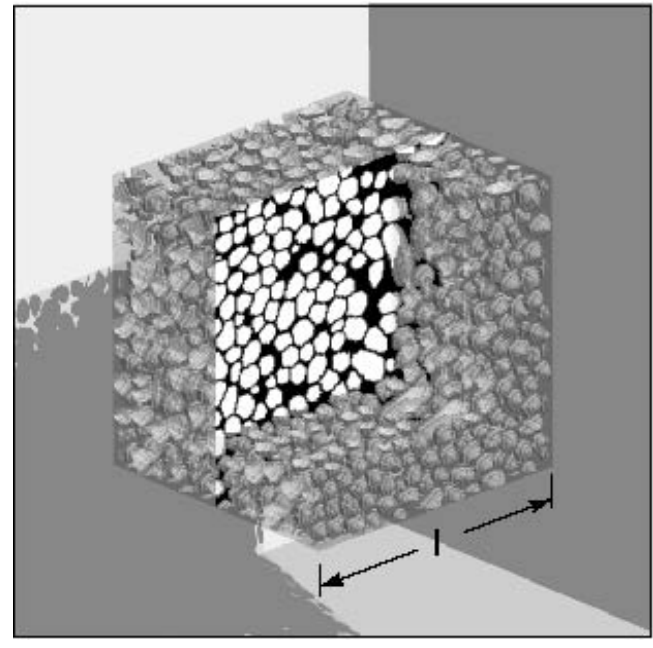

Figure 9. A complex unit cell containing realistic biofilm structure.

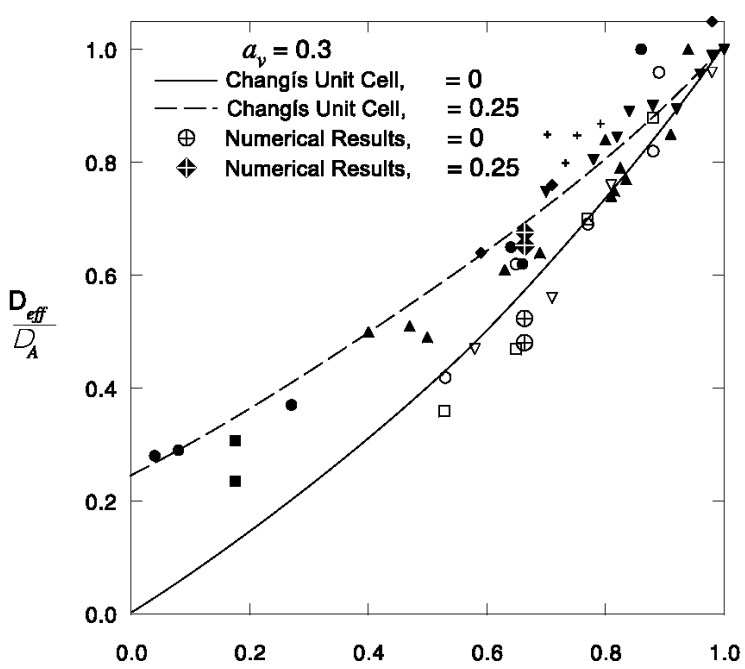

Figure 10. Comparison of experimental data, the analytical solution for the simple unit cell, and the numerical solution to the complex unit cell. 